\title{
Numerical solution of Boussinesq systems of the Bona-Smith family
}

\author{
D. C. Antonopoulos ${ }^{a}$, V. A. Dougalis ${ }^{\mathrm{a}, \mathrm{b}, 1}$ and D. E. Mitsotakis ${ }^{\mathrm{c}}$ \\ ${ }^{a}$ Department of Mathematics, University of Athens, 15784 Zographou, Greece \\ ${ }^{\mathrm{b}}$ Institute of Applied and Computational Mathematics, FO.R.T.H., P.O. Box 1527, 70013 Heraklion, Greece \\ ${ }^{\mathrm{c}}$ UMR de Mathématiques, Université de Paris-Sud, Bâtiment 425, P.O. Box, 91405 Orsay France
}

\begin{abstract}
In this paper we consider the one-parameter family of Bona-Smith systems, which belongs to the class of Boussinesq systems modelling two-way propagation of long waves of small amplitude on the surface of water in a channel. We study numerically three initial-boundary-value problems for these systems, corresponding, respectively, to homogeneous Dirichlet, reflection, and periodic boundary conditions posed at the endpoints of a finite spatial interval. We approximate these problems using the standard Galerkin-finite element method for the spatial discretization and a fourth-order, explicit Runge-Kutta scheme for the time stepping, and analyze the convergence of the fully discrete schemes. We use these numerical methods as exploratory tools in a series of numerical experiments aimed at illuminating interactions of solitary-wave solutions of the Bona-Smith systems, such as head-on and overtaking collisions, and interactions of solitary waves with the boundaries.
\end{abstract}

Key words: Water waves, Boussinesq approximation, Bona-Smith systems, initial-boundary value problems, solitary waves, numerical methods, error estimates, fully discrete Galerkin-finite element methods

\section{Introduction}

We consider the following family of Boussinesq type systems of water wave theory, introduced by Bona and Smith in [12],

$$
\begin{aligned}
& \eta_{t}+u_{x}+(\eta u)_{x}-b \eta_{x x t}=0, \\
& u_{t}+\eta_{x}+u u_{x}+c \eta_{x x x}-b u_{x x t}=0,
\end{aligned}
$$

\footnotetext{
Email addresses: antonod@math.uoa.gr (D. C. Antonopoulos), doug@math.uoa.gr (V. A. Dougalis), dimitrios.mitsotakis@math.u-psud.fr (D. E. Mitsotakis).

1 Corresponding author
} 
for $x \in \mathbb{R}, t>0$, where $b>0, c \leq 0$. (For modelling purposes, [7], the constants $b$ and $c$ are of the form $b=\left(3 \theta^{2}-1\right) / 6, c=\left(2-3 \theta^{2}\right) / 3$, where $\theta^{2} \in[2 / 3,1]$, cf. [7].) The system is supplemented by initial conditions of the form

$$
\eta(x, 0)=\eta_{0}(x), u(x, 0)=u_{0}(x),
$$

where $\eta_{0}, u_{0}$ are given functions on $\mathbb{R}$.

The systems (1) are Boussinesq approximations of the two-dimensional Euler equations and model two-way propagation of long waves of small amplitude (when the Stokes number is of $O(1)$ ) of an incompressible, inviscid fluid in a uniform horizontal channel of finite depth with a free surface. The variables in (1) are nondimensional and unscaled: $x$ and $t$ are proportional to position along the channel and time, respectively, while $\eta(x, t)$ and $u(x, t)$ are proportional to the deviation of the free surface above an undisturbed level, and to the horizontal velocity of the fluid at a height $y=-1+\theta(1+\eta(x, t))$, respectively. In terms of these variables the bottom of the channel is at $y=-1$, while the free surface corresponds to $\theta=1$.

The well-posedness of the initial-value problem (1)-(2) has been studied in [12] and [8]. For $b>0, c<0$ the problem is globally well posed in appropriate pairs of Sobolev spaces or in spaces of bounded, continuously differentiable functions, provided $\inf _{x \in \mathbb{R}} \eta_{0}(x)>-1$ and under one additional mild restriction on the initial data. If $b>0, c=0$ (e.g. when $\theta^{2}=2 / 3$ ), one obtains the BBM-BBM system whose Cauchy problem is well posed only locally in time.

In [4] the present authors studied the well-posedness of three types of initial-boundary-value problems (ibvp's) for the Bona-Smith systems (1) on bounded spatial intervals $[-L, L]$, in which (1) and the initial conditions $\eta(x, 0)=\eta_{0}(x), u(x, 0)=u_{0}(x), x \in[-L, L]$, are supplemented by three types of boundary conditions posed at $x= \pm L$ for $t \geq 0$ : (i) Nonhomogeneous Dirichlet boundary conditions, wherein $\eta$ and $u$ are given functions of $t$ at $x= \pm L$. (ii) Reflection boundary conditions, i.e. $\eta_{x}=0, u=0$ at $x= \pm L$ for all $t \geq 0$. (iii) Periodic boundary conditions on $\eta$ and $u$ at $x= \pm L$. It was proved that in appropriate pairs of spaces, the ibvp with Dirichlet boundary conditions is locally well posed, while the ibvp's with reflection of periodic boundary conditions are globally well posed under restrictions on the initial data similar to those imposed in the case of the Cauchy problem. (A local well-posedness result for the ibvp with Dirichlet end conditions of the BBM-BBM system had been previously established by Bona and Chen in [6].)

Our first aim in this paper is to derive and analyze rigorously fully discrete numerical methods for approximating the solutions of these three ibvp's for the Bona-Smith systems. We shall use the standard Galerkin-finite element method with piecewise polynomial functions in $x$ and the classical, fourth-order Runge-Kutta method for time-stepping. To describe the results, we introduce now function spaces that we will employ in the sequel. Let $I=(-L, L)$. For a nonnegative integer $k$, we let $C^{k}=C^{k}(\bar{I})$ be the space of $k$ times continuously differentiable real-valued functions on $\bar{I}$ equipped with the norm $\|v\|_{C^{k}}:=\max _{0 \leq j \leq k} \max _{x \in \bar{I}}\left|v^{(j)}(x)\right| .\left(C_{0}^{k}\right.$ will denote the functions in $C^{k}$ which vanish at $x= \pm L$.) We also let $H^{k}=H^{k}(I)$ be the usual (Hilbert) Sobolev spaces of realvalued functions on $I$ having generalized derivatives of order up to $k$ in $L^{2}=L^{2}(I)$. The norm on $H^{k}$ will be given by $\|v\|_{k}:=\left(\sum_{j=0}^{k}\left\|v^{(j)}\right\|^{2}\right)^{1 / 2}$, where $\|\cdot\|$ denotes the norm on $L^{2} \equiv H^{0}$. The $L^{2}$ inner product will be denoted by $(\cdot, \cdot)$, while $H_{0}^{1}$ will denote the subspace of $H^{1}$ whose elements vanish at $x= \pm L$. On occasion we shall also use the spaces $L^{\infty}=L^{\infty}(I)$ and $W_{\infty}^{1}=W_{\infty}^{1}(I)$ whose usual norms will be denoted by $\|\cdot\|_{L^{\infty}},\|\cdot\|_{W_{\infty}^{1}}$, respectively.

Rigorous error estimates for Bona-Smith and BBM-BBM type systems were previously proved in [39], [27], [6], [13]. Winther, in [39], considered the homogeneous Dirichlet ibvp for the Bona-Smith system with $\theta^{2}=1$, which he discretized in space using a nonstandard Galerkin-finite element method (a combination of an $H^{1}$ method and a standard one, with polynomials of different degree for the approximation of $\eta$ and $u$ ); he proved optimal-order error estimates for the semidiscrete 
approximations of $\eta$ and $u$ in a variety of norms. Pelloni, in [27], considered the periodic ivp for the same system, which she discretized in space using the spectral Galerkin method proving convergence of the semidiscrete scheme. Numerical experiments with spectral methods (discretized in time with the classical fourth-order Runge-Kutta scheme) on various Boussinesq systems of the form (1) were also reported in [27]; cf. also [28]. In [6], Bona and Chen considered the BBM-BBM system with nonhomogeneous Dirichlet boundary conditions that they discretized using a fully discrete finite difference scheme of fourth-order of accuracy in space and time based on the numerical integration of the integral representation of the spatial operator $\left(I-\frac{1}{6} \partial_{x}^{2}\right)^{-1}$ and on a multistep predictorcorrector method in time. They proved optimal-order error estimates for their numerical method and used it in a thorough experimental investigation of various properties of solitary wave solutions of the BBM-BBM system, and their interactions. In [13], Chatzipantelidis discretized the BBMBBM system in the case of homogeneous Dirichlet boundary conditions using the standard Galerkin method in space and high order explicit multistep schemes in time; he proved optimal-order $L^{2}$ error estimates for the resulting fully discrete schemes.

In Section 2 of the present paper we consider the three ibvp's (i)-(iii) for the whole family of the Bona-Smith systems. The ibvp's with homogeneous (for simplicity) Dirichlet and reflection boundary conditions are discretized in space by the standard Galerkin-finite element method, using, in general, finite-dimensional subspaces of $C^{\mu}$, for $\mu \geq 1$, that consist of piecewise polynomial functions of degree $r-1$, where $r \geq \mu+2$. The approximations of both $\eta$ and $u$ are sought in the same subspace. Due to the presence of the odd-order derivative in the term $\eta_{x x x}$ for $c \neq 0$ and the non-periodic boundary conditions, the standard Galerkin semidiscretization is not expected to converge at the optimal rate in $L^{2}$, cf. e.g. [22]. In Section 2 we prove that the semidiscrete approximation of $\eta$ converges at the optimal rates $r-2$, respectively $r-1$, in $H^{2}$, respectively $W_{\infty}^{1}$, norms, while the analogous approximation of $u$ converges at the rates $r-2$, respectively $r-1$, in the $H^{1}$, respectively $L^{\infty}$, norms. Numerical experiments in Paragraph 3.1 suggest that both approximations converge suboptimally, i.e. at the rate $r-1$, in $L^{2}$. Despite this drawback, the standard Galerkin method always yields a symmetric, positive definite mass matrix and, therefore, a semidiscretization that is always well defined, at least locally in time, for any system of the form (1) with $b \geq 0$. In addition, in the case of the periodic boundary conditions, it is proved, in Paragraph 2.5, that when subspaces of smooth splines are used on a uniform mesh, the resulting standard Galerkin semidiscretization is of optimal order (e.g. $r$ in $L^{2}$ ) for all Bona-Smith systems, as in the case of linear p.d.e.'s, [35], or the KdV equation, [5].

Due, essentially, to the positive coefficients $b$ of the $-\eta_{x x t}$ and $-u_{x x t}$ terms in both equations of the system (1), it is shown, in Paragraph 2.2, that the systems of ordinary differential equations (ode's) that represent the standard Galerkin semidiscretization of (1) are not stiff. As a consequence, an explicit time stepping method may be used for their discretization without imposing stringent stability conditions on the time step in terms of the spatial meshlength. In this paper we use the classical, explicit, four-stage, fourth-order accurate Runge-Kutta scheme, which is shown to be unconditionally stable and to lead to full discretizations that are indeed fourth-order accurate in time in various norms for all three ibvp's considered, and require only linear systems to be solved at each time step. (Of course, other Boussinesq systems lead to stiff systems. For example, the so-called $\mathrm{KdV}-\mathrm{KdV}$ system, with coefficients obtained if we put $b=0, c=1 / 6$ and add a term of the form $\frac{1}{6} \eta_{x x x}$ in the first equation of (1), leads to very stiff Galerkin semidiscretizations that have to be integrated in time by e.g. $B$-stable, implicit Runge-Kutta schemes, cf. [10], to avoid severe stability restrictions on the time step. Such schemes, being implicit, lead to nonlinear algebraic systems that must be solved at each time step.)

Our second goal in this paper is to use the aforementioned fully discrete Galerkin method (with, mainly, $C^{2}$ cubic splines for the spatial discretization) in a series of numerical experiments aimed at illuminating phenomena of interactions between solitary-wave solutions of the Bona-Smith systems 
and interactions of solitary waves with the boundary of the computational domain.

The (classical) solitary waves are travelling wave solutions of the system (1) of the form $\eta_{s}(x, t)=$ $\eta_{s}\left(x+x_{0}-c_{s} t\right), u_{s}(x, t)=u_{s}\left(x+x_{0}-c_{s} t\right)$, where $x_{0} \in \mathbb{R}$ and the speed $c_{s}$ is constant. The functions $\eta_{s}=\eta_{s}(\xi), u_{s}=u_{s}(\xi), \xi \in \mathbb{R}$, will be assumed to be smooth, positive, even, and decaying monotonically to zero, along with their derivatives, as $\xi \rightarrow \pm \infty$. Substituting the travelling wave expressions into (1), integrating once, and setting the integration constants equal to zero, we obtain the system of second-order nonlinear ode's

$$
\begin{aligned}
& -c_{s} \eta+u+\eta u+b c_{s} \eta^{\prime \prime}=0, \\
& -c_{s} u+\eta+\frac{1}{2} u^{2}+c \eta^{\prime \prime}+b c_{s} u^{\prime \prime}=0,
\end{aligned}
$$

where we denote $\eta_{s}(\xi), u_{s}(\xi)$ simply by $\eta(\xi), u(\xi)$ and ${ }^{\prime}=\frac{d}{d \xi}$. (Note that if $(\eta, u)$ is a solution of (3) for some $c_{s}>0$, then $(\eta,-u)$ is also a solution propagating with speed $-c_{s}$, i.e. to the left, as $t$ increases. In the sequel we will assume that $c_{s}>0$.)

Existence of solutions of the ode system (3) was established by Toland, [36], in the case $\theta^{2}=1$ for any value of the speed $c_{s}>1$. Subsequently, Toland, [38], proved existence of solutions of a more general system of second-order ode's; this theory was applied by Chen, [15], to establish existence of solitary waves for several Boussinesq systems, including the BBM-BBM system, and also in [20], where the existence of solitary waves of the whole family of Bona-Smith systems was proved for all $c_{s}>1$. It is also possible to prove uniqueness of the classical solitary waves, in general for restricted ranges of the speed $c_{s}$. See e.g. [37] for the case $\theta^{2}=1$ and [20] for the extension of the theory to systems with $\theta^{2} \in(0.816,1]$. For a review of solitary-wave solutions of more general Boussinesq systems we refer the reader to [25] and [21].

The importance of solitary waves lies mainly in the distinguished role they apparently play in the evolution and long-time asymptotics of solutions of the Cauchy problem for (1) that develop from arbitrary initial data that decays suitably at infinity. In particular, such initial data is resolved as time increases into a series of solitary waves plus small-size, oscillatory dispersive tails. This property has been rigorously proved for integrable equations, such as the KdV equation, and has been observed numerically in many other examples of nonlinear dispersive equations, and in particular in the case of Boussinesq systems, cf. e.g. [6], [28], [1], [18]. It is related to the fact that the solitary waves of the systems appear to be stable under small perturbations. As explained e.g in [26], [18], the classical variational theory for studying the orbital stability of solitary waves fails in the case of Boussinesq (and other) systems. Still missing is also a rigorous proof of their asymptotic stability. In [26], Pego and Weinstein have analyzed the linearized 'convective' asymptotic stability of the solitary waves of some Boussinesq type fourth-order p.d.e.'s and of the 'classical' Boussinesq system. A detailed numerical study of various stability properties of the solitary waves of the Bona-Smith systems was carried out in [18] using the fully discrete Galerkin scheme analyzed here for solving the periodic initial-value problem. One of the outcomes of the numerical experiments of [18] is that the solitary waves of (1) appear to be asymptotically stable under a variety of perturbations.

In this paper, we first compare, in Paragraph 3.2, numerically computed amplitude $\left(A_{s}\right)$ vs. speed $\left(c_{s}\right)$ data for the solitary waves of the Bona-Smith systems with analogous available experimental and analytical data for the solitary waves of the Euler equations. Our conclusion is that the points representing the pairs $\left(A_{s}, c_{s}\right)$ for the Bona-Smith systems for various values of $\theta^{2}$ remain close to Scott Russell's prediction $c_{s}=\sqrt{1+A_{s}}$, that he made by fitting his experimental observations, [30].

In Paragraph 3.3 we study numerically interactions between solitary waves of the systems. The interactions are of two kinds, namely head-on and overtaking collisions. Head-on collisions were studied in detail in the context of the BBM-BBM system in [6]. Particular emphasis was placed in [6] in comparing the computed maximum amplitude during the collision, and the accompanying 
phase shift, with available analytical approximations of these quantities valid for the Euler equations, [32]; fairly good agreement was observed. Our computations with other systems of the Bona-Smith family yield, in general, qualitatively similar results. The main difference is that if $\theta^{2} \neq 2 / 3$, the maximum amplitude after the collision apparently decreases monotonically to a value smaller than its value before the collision, and this loss of amplitude appears to be permanent. In contrast to this, in the case of the BBM-BBM system the maximum amplitude returns to its pre-collision level after some transient variation.

In Paragraph 3.3.2 we study numerically overtaking collisions of solitary waves of the Bona-Smith systems that travel in the same direction with different speeds. Such interactions of course have been studied in detail for one-way models, by the inverse scattering transform in the case of integrable equations like the KdV, [24], or numerically for nonintegrable equations, [11]. They have recently been studied in detail by experimental and numerical means in the case of the full Euler equations by Craig et al. in [16]. Our numerical computations yield qualitatively similar results with those of these references. We also study in some detail a small wavelet structure generated during the overtaking collision and moving to a direction opposite to that of the solitary waves.

Finally, in Paragraph 3.4, we study numerically some aspects of the interaction of solitary waves of the Bona-Smith systems with the boundary of the computational domain. Specifically, we consider 'collisions' of solitary waves with the endpoints of an interval, where either reflection or homogeneous Dirichlet boundary conditions have been posed. The case of a solitary wave impinging on a reflection boundary is of course equivalent to (one half of) a head-on collision of the solitary wave with its symmetric image. In this case, as was mentioned already, the solitary waves of the systems are reflected backwards with a tiny permanent loss of amplitude and are followed by a dispersive tail of very small amplitude. The interaction with a homogeneous Dirichlet boundary is more complicated and we describe it in some detail

Most of the theoretical results of Section 2 were first proved in [1]. Some were announced in preliminary form in [2], [3], and [18]. The proofs of some results of the paper that are stated here without proof are available in electronic form upon request to the authors.

\section{Numerical methods and error estimates}

\subsection{Preliminaries}

Let $-L=x_{0}<x_{1}<\cdots<x_{J+1}=L$ denote a partition of the interval $\bar{I}=[-L, L]$, which is quasiuniform, in the sense that for some positive constant $c, \min _{j}\left(x_{j+1}-x_{j}\right) \geq c h, h:=\max _{j}\left(x_{j+1}-\right.$ $x_{j}$ ). Given integers $r \geq 2$ and $0 \leq \mu \leq r-2$, we shall consider the finite dimensional spline spaces $S_{h}=S_{h}(\mu, r)=\left\{\phi \in C^{\mu}:\left.\phi\right|_{\left[x_{j}, x_{j+1}\right]} \in \mathbb{P}_{r-1}, 0 \leq j \leq J\right\}$, and $S_{h}^{0}=S_{h} \cap\{\phi \in C(\bar{I}): \phi(-L)=$ $\phi(L)=0\}$, where $\mathbb{P}_{k}$ denotes the space of polynomials of degree at most $k$. Hence $S_{h}$ is a subspace of $H^{\mu+1}$ and $S_{h}^{0}$ of $H^{\mu+1} \cap H_{0}^{1}$. It is well-known, cf. e.g. [17], [31], that $S_{h}$ and $S_{h}^{0}$ have the following approximation properties:

Lemma 1 Let $m, k$ be integers such that $0 \leq m \leq \mu+1$ and $m<k \leq r$. Then, there exists a constant $C$, independent of $h$, such that

$$
\min _{\chi \in S_{h}}\left\|(w-\chi)^{(m)}\right\| \leq C h^{k-m}\left\|w^{(k)}\right\|, \quad \text { if } w \in H^{k},
$$

and

$$
\min _{\chi \in S_{h}}\left\|(w-\chi)^{(m)}\right\|_{L^{\infty}} \leq C h^{k-m}\left\|w^{(k)}\right\|_{L^{\infty}}, \text { if } w \in C^{k} .
$$

Both results hold in the case of $S_{h}^{0}$ as well, when $w \in H^{k} \cap H_{0}^{1}$ and $w \in C_{0}^{k}$, respectively. 
In the sequel we shall use two types of elliptic projections onto the spline spaces. We define the linear mapping $R_{h}: H^{1} \rightarrow S_{h}$ by $a_{N}\left(R_{h} v, \chi\right)=a_{N}(v, \chi), \forall \chi \in S_{h}$, where $a_{N}: H^{1} \times H^{1} \rightarrow \mathbb{R}$ is the bilinear form $a_{N}(v, w):=(v, w)+b\left(v^{\prime}, w^{\prime}\right)$. Denoting by $a_{D}$ the restriction of $a_{N}$ to $H_{0}^{1} \times H_{0}^{1}$, we also define the elliptic projection $R_{h}^{0}: H_{0}^{1} \rightarrow S_{h}^{0}$ by $a_{D}\left(R_{h}^{0} w, \chi\right)=a_{D}(w, \chi), \forall \chi \in S_{h}^{0}$. Since $a_{N}$ and $a_{D}$ are coercive in their respective domains of definition, it follows that the mappings $R_{h}$ and $R_{h}^{0}$ are well-defined. The following lemma summarizes some of their stability properties that will be used in the sequel. (By $C$ we shall denote generic constants independent of $h$.)

Lemma 2 (i) $R_{h}$ and $R_{h}^{0}$ are stable in $L^{\infty}$, in the sense that there exist constants $C$ such that

$$
\left\|R_{h} v\right\|_{L^{\infty}} \leq C\|v\|_{L^{\infty}}, \forall v \in H^{1}, \quad\left\|R_{h}^{0} v\right\|_{L^{\infty}} \leq C\|v\|_{L^{\infty}}, \forall v \in H_{0}^{1} .
$$

(ii) If $\mu \geq 1$, then $R_{h}$ and $R_{h}^{0}$ are stable in $H^{2}$, in the sense that there exist constants $C$ such that

$$
\left\|R_{h} v\right\|_{2} \leq C\|v\|_{2} \forall v \in H^{2}, \quad\left\|R_{h}^{0} v\right\|_{2} \leq C\|v\|_{2} \forall v \in H^{2} \cap H_{0}^{1} .
$$

(iii) If $\mu \geq 1$, then $R_{h}$ and $R_{h}^{0}$ are stable in $W_{\infty}^{1}$, in the sense that there exist constants $C$ such that

$$
\left\|R_{h} v\right\|_{W_{\infty}^{1}} \leq C\|v\|_{W_{\infty}^{1}} \forall v \in W_{\infty}^{1}, \quad\left\|R_{h}^{0} v\right\|_{W_{\infty}^{1}} \leq C\|v\|_{W_{\infty}^{1}} \forall v \in W_{\infty}^{1} \cap H_{0}^{1} .
$$

PROOF. The stability of $R_{h}^{0}$ in $L^{\infty}$ is proved in [34]. The proof of the stability of $R_{h}$ in $L^{\infty}$ follows along similar lines. The proofs of (ii) and (iii) are standard and may be found in detail e.g. in [1].

From Lemmata 1 and 2 there follows in a standard way

Lemma 3 If $\mu \geq 1$, then

(i) $\left\|R_{h}^{0} v-v\right\|_{k} \leq C h^{r-k}\|v\|_{r}$, if $v \in H^{r} \cap H_{0}^{1}$ and $k=0,1,2$.

(ii) $\left\|R_{h}^{0} v-v\right\|_{W_{\infty}^{k}} \leq C h^{r-k}\|v\|_{W_{\infty}^{r}}$, if $v \in C_{0}^{r}$ and $k=0,1$.

Analogous estimates to (i) and (ii) hold for $R_{h}$ if $v \in H^{r}$ and $v \in C^{r}$, respectively.

\subsection{Semidiscretization of the ibvp with homogeneous Dirichlet boundary conditions}

We consider, for simplicity, the homogeneous Dirichlet ibvp for the Bona-Smith systems, that we rewrite here for the convenience of the reader. For $b>0, c<0$ we seek $\eta$ and $u$ such that

$$
\begin{aligned}
& \eta_{t}+u_{x}+(\eta u)_{x}-b \eta_{x x t}=0, \\
& u_{t}+\eta_{x}+u u_{x}+c \eta_{x x x}-b u_{x x t}=0, \quad x \in \bar{I}, t \geq 0, \\
& u(x, 0)=u_{0}(x), \eta(x, 0)=\eta_{0}(x), x \in \bar{I}, \\
& \eta( \pm L, t)=u( \pm L, t)=0, t \geq 0 .
\end{aligned}
$$

We let $\mu \geq 1$ and $r \geq \mu+2$. Then, the standard Galerkin semidiscretization of (D) in $S_{h}^{0}=S_{h}^{0}(\mu, r)$ is defined as follows. Let $[0, T]$ be the maximal interval of existence and uniqueness of the solution of (D) in an appropriate function space to be specified below. We seek $\eta_{h}, u_{h}:[0, T] \rightarrow S_{h}^{0}$ satisfying for $0 \leq t \leq T$

$$
\begin{aligned}
& a_{D}\left(\eta_{h t}, \chi\right)+\left(\left(u_{h}+\eta_{h} u_{h}\right)_{x}, \chi\right)=0, \forall \chi \in S_{h}^{0}, \\
& a_{D}\left(u_{h t}, \psi\right)+\left(\eta_{h_{x}}, \psi\right)-c\left(\eta_{h_{x x}}, \psi^{\prime}\right)+\left(u_{h} u_{h x}, \psi\right)=0, \forall \psi \in S_{h}^{0},
\end{aligned}
$$

with initial conditions

$$
\eta_{h}(0)=R_{h}^{0} \eta_{0}, \quad u_{h}(0)=R_{h}^{0} u_{0} .
$$


(It is to be noted that other types of suitable approximations of $\eta_{0}$ and $u_{0}$ in $S_{h}^{0}$, e.g. $L^{2}$ projections, interpolants etc., may be used as initial conditions without affecting the error estimates. We have used the elliptic projections for simplicity.) We represent (4)-(5) more compactly by defining the mappings $\hat{f}: L^{2} \rightarrow S_{h}^{0}$ by $a_{D}(\hat{f}(w), \chi)=\left(w, \chi^{\prime}\right), \forall \chi \in S_{h}^{0}, \hat{g}: H^{2} \rightarrow S_{h}^{0}$ by $\hat{g}(v)=c \hat{f}\left(v^{\prime \prime}\right)+\hat{f}(v)$, $F: H^{1} \times H^{1} \rightarrow S_{h}^{0}$ by $F(v, w)=\hat{f}(v w), f: H^{1} \times H^{1} \rightarrow S_{h}^{0}$ by $f(v, w)=\hat{f}(w)+F(v, w)$, $g: H^{2} \times H^{1} \rightarrow S_{h}^{0}$ by $g(v, w)=\hat{g}(v)+\frac{1}{2} F(w, w)$. Then, we may write (4)-(5) as

$$
\begin{aligned}
& \eta_{h_{t}}=f\left(\eta_{h}, u_{h}\right), \quad 0 \leq t \leq T, \\
& u_{h t}=g\left(\eta_{h}, u_{h}\right), \\
& \eta_{h}(0)=R_{h}^{0} \eta_{0}, u_{h}(0)=R_{h}^{0} u_{0} .
\end{aligned}
$$

Choosing a basis for $S_{h}^{0}$, we may see that (6) represents an initial-value problem for the system of o.d.e.'s satisfied by the coefficients of $\eta_{h}$ and $u_{h}$ with respect to this basis. Since $f$ and $g$ are continuous and locally Lipschitz (6) possesses a unique solution at least in an interval $\left[0, t_{h}\right)$. In the error estimate of Proposition 5 we shall show in particular that this solution may be extended up to $t=T$. We first establish some key properties of the mapping $\hat{f}$.

Lemma 4 There exists a constant $C$ independent of $h$ such that

$$
\begin{aligned}
& \text { (i) }\|\hat{f}(v)\|_{1} \leq C\|v\|, v \in L^{2}, \quad \text { (ii) }\|\hat{f}(v)\|_{2} \leq C\|v\|_{1}, v \in H^{1}, \\
& \text { (iii) }\|\hat{f}(v)\|_{W_{\infty}^{1}} \leq C\|v\|_{L^{\infty}}, v \in L^{\infty}, \text { (iv) }\left\|\hat{f}\left(v^{\prime}\right)\right\|_{L^{\infty}} \leq C\|v\|_{L^{\infty}}, v \in H^{1} .
\end{aligned}
$$

PROOF. The inequality (i) follows immediately from the definition of $\hat{f}$. To prove (ii) consider the bvp $w-b w^{\prime \prime}=-v^{\prime}$ for $v \in H^{1}$, with $w( \pm L)=0$. Then $a_{D}(w, \chi)=a_{D}\left(R_{h}^{0} w, \chi\right)=\left(v, \chi^{\prime}\right)$ for $\chi \in S_{h}^{0}$, whence $R_{h}^{0} w=\hat{f}(v)$, and (ii) follows in view of (ii) of Lemma 2. In order to prove (iii) consider the bvp $\gamma-b \gamma^{\prime \prime}=-w, \gamma( \pm L)=0$, where, given $v \in L^{\infty}, w(x):=\frac{1}{b} \int_{-L}^{x} v d \xi-\frac{(x+L)}{2 b L} \int_{-L}^{L} v d \xi, x \in \bar{I}$. It follows that $w \in W_{\infty}^{1} \cap H_{0}^{1}$ and $\|w\|_{W_{\infty}^{1}} \leq C\|v\|_{L^{\infty}}$. In addition, it is straightforward to verify that for $\chi \in S_{h}^{0}, a_{D}\left(\hat{f}(v)-R_{h}^{0} w, \chi\right)=a_{D}\left(R_{h}^{0} \gamma, \chi\right)$. It follows that $\hat{f}(v)=R_{h}^{0}(w+\gamma)$, from which (iii) follows in view of the above and (iii) of Lemma 2. Finally, consider the bvp $\gamma-b \gamma^{\prime \prime}=-w$, $\gamma( \pm L)=0$, where, for $v \in H^{1}, w(x):=\frac{1}{b}(v(x)-v(-L))-\frac{(x+L)}{2 b L}(v(L)-v(-L)), x \in \bar{I}$, so that $w \in H_{0}^{1}$ and $\|w\|_{L^{\infty}} \leq C\|v\|_{L^{\infty}}$. As before, we may check that $\hat{f}\left(v^{\prime}\right)=R_{h}^{0}(w+\gamma)$, from which (iv) follows in view of (i) of Lemma 2.

We can now prove an error estimate in $H^{2} \times H^{1}$ for the semidiscrete approximation. In what follows, $C(0, T ; X)$ denotes the continuous maps from $[0, T]$ onto the Banach space $X$.

Proposition 5 Let $T>0$ be such that the classical solution $(\eta, u)$ of $(D)$ lies in $C\left(0, T ; H^{r} \cap\right.$ $\left.H_{0}^{1}\right) \times C\left(0, T ; H^{r-1} \cap H_{0}^{1}\right)$. Then, if $h$ is sufficiently small, the semidiscrete problem (6) has a unique solution $\left(\eta_{h}, u_{h}\right)$ on $[0, T]$, which satisfies, for some constant $C$ independent of $h$,

$$
\left\|\eta(t)-\eta_{h}(t)\right\|_{2}+\left\|u(t)-u_{h}(t)\right\|_{1} \leq C h^{r-2}, \quad 0 \leq t \leq T .
$$

PROOF. Let $\tilde{M}>0$ be a constant large enough so that

$$
\max _{0 \leq t \leq T}\left(\|\eta(t)\|_{2}+\|u(t)\|_{1}\right) \leq \tilde{M}
$$

and $\left\|R_{h}^{0} \eta_{0}\right\|_{2}+\left\|R_{h}^{0} u_{0}\right\|_{1} \leq 3 \tilde{M} / 2$ (cf. Lemma 2 ). Let $0<t_{h} \leq T$ be the maximal time for which there holds

$$
\left\|u_{h}(t)\right\|_{1} \leq 2 \tilde{M}, \quad 0 \leq t \leq t_{h} .
$$


Define $\rho:=\eta-R_{h}^{0} \eta, \theta:=R_{h}^{0} \eta-\eta_{h}, \sigma:=u-R_{h}^{0} u, \xi:=R_{h}^{0} u-u_{h}$. Then, by (6) and (D) we have

$$
\begin{aligned}
\theta_{t} & =\hat{f}(\xi+\sigma)+F(\eta, \sigma+\xi)+F\left(\rho+\theta, u_{h}\right), \\
\xi_{t} & =\hat{g}(\theta+\rho)+\frac{1}{2}\left[F(u, \sigma+\xi)+F\left(\sigma+\xi, u_{h}\right)\right] .
\end{aligned}
$$

Therefore, using Lemma 3, (i) and (ii) of Lemma 4 and (9) we conclude that there exists a constant $C=C(\eta, u, \tilde{M})$ such that for $0 \leq t \leq t_{h}$

$$
\begin{aligned}
&\left\|\theta_{t}\right\|_{2} \leq C\left(h^{r-2}+\|\theta\|_{1}+\|\xi\|_{1}\right), \\
&\left\|\xi_{t}\right\|_{1} \leq C\left(h^{r-2}+\|\theta\|_{2}+\|\xi\|_{1}\right) .
\end{aligned}
$$

Using these estimates there follows for $0 \leq t \leq t_{h}$

$$
\|\theta(t)\|_{2}+\|\xi(t)\|_{1} \leq\|\theta(0)\|_{2}+\|\xi(0)\|_{1}+C\left[h^{r-2}+\int_{0}^{t}\left(\|\theta(\tau)\|_{2}+\|\xi(\tau)\|_{1}\right) d \tau\right],
$$

which, by Gronwall's lemma, since $\theta(0)=\xi(0)=0$, yields for some constant $C=C(\eta, u, \tilde{M}, T)$

$$
\|\theta(t)\|_{2}+\|\xi(t)\|_{1} \leq C h^{r-2}, \quad 0 \leq t \leq t_{h} .
$$

This implies by (8), since $\|\sigma(t)\|_{1} \leq C h^{r-2}$ and $u_{h}=u-(\sigma+\xi)$, that $\left\|u_{h}(t)\right\|_{1} \leq C h^{r-2}+\tilde{M}$. Hence, since $r \geq 3$, for $h$ sufficiently small we have that $\left\|u_{h}(t)\right\|_{1}<2 \tilde{M}$ for $t \in\left[0, t_{h}\right]$ contradicting the definition of $t_{h}$. Therefore, (9) and (14) hold up to $t=T$ and the result follows from Lemma 3 .

The following proposition is the main result of this section:

Proposition 6 Suppose that for the classical solution of $(D)$ there holds that $(\eta, u) \in C\left(0, T ; C_{0}^{r}\right) \cap$ $C\left(0, T ; C_{0}^{r-1}\right)$ for some $0<T<\infty$. Then, for $h$ sufficiently small, there exists a constant $C$ independent of $h$, such that for $0 \leq t \leq T$

$$
\left\|\eta(t)-\eta_{h}(t)\right\|_{W_{\infty}^{1}}+\left\|u(t)-u_{h}(t)\right\|_{L^{\infty}} \leq C h^{r-1} .
$$

PROOF. From (10) and (11), using Lemma 3(ii) and Lemma 4(iii) and (iv), we have $\left\|\theta_{t}\right\|_{W_{\infty}^{1}} \leq$ $C\left(h^{r}+\|\theta\|_{L^{\infty}}+\|\xi\|_{L^{\infty}}\right)$, and $\left\|\xi_{t}\right\|_{L^{\infty}} \leq C\left(h^{r-1}+\|\theta\|_{W_{\infty}^{1}}+\|\xi\|_{L^{\infty}}\right)$. The result now follows by Gronwall's lemma.

Remark 7 The error estimates (7) and (15) give optimal rates of convergence for $\eta-\eta_{h}$ in $H^{2}$ and $W_{\infty}^{1}$ and suboptimal rates for $u-u_{h}$ in $H^{1}$ and $L^{\infty}$. In the numerical experiments of Section 3.1, where smooth cubic splines are used for the spatial discretization (i.e. $\mu=2, r=4$ ), the optimal rates for $\eta-\eta_{h}$ are confirmed, while it is observed that the rate of convergence of $\left\|u-u_{h}\right\|_{L^{\infty}}$ is equal to three. Thus, it seems that the exponent $r-1$ in (15) is sharp. The observed rates of convergence in $L^{2}$ for both components of the error are equal to 3. This suboptimal rate is expected (see the remarks in the Introduction) for the standard Galerkin method in the presence of non-periodic boundary conditions due to the term $\eta_{x x x}$ in the Boussinesq system at hand.

Remark 8 In the case of the BBM-BBM systems $(b>0, c=0)$ it is straightforward (cf. e.g. [13]) to show that optimal-order estimates hold for $\eta-\eta_{h}$ and $u-u_{h}$ in $L^{2}\left(o f O\left(h^{r}\right)\right.$ ) and in $H^{1}$ (of $O\left(h^{r-1}\right)$ ), even for subspaces with $\mu=0$ and $r \geq 2$.

Remark 9 Using e.g. the estimates (12), (13), the inequalities in the proof of Proposition 6, and the properties of the elliptic projection operators, one obtains similar estimates for the temporal derivatives of the errors $\eta-\eta_{h}$ and $u-u_{h}$. It is also not hard to show (using the fact that 
$\max _{0 \leq t \leq T}\left(\left\|\eta_{h}\right\|_{2}+\left\|u_{h}\right\|_{1}\right) \leq C$, where $C$ is independent of $h$, a byproduct of the proof of Proposition 5) that temporal derivatives of all orders of $\eta_{h}$ and $u_{h}$ are bounded on $[0, T]$ in the $H^{2}$ and $H^{1}$ norms, respectively, by constants independent of $h$. For example, repeated applications of (i) and (ii) of Lemma 4 to the formulas $\eta_{h_{t}}=f\left(\eta_{h}, u_{h}\right), u_{h t}=g\left(\eta_{h}, u_{h}\right)$ yield $\left\|\eta_{h_{t}}\right\|_{2}+\left\|u_{h t}\right\|_{1} \leq C$ on $[0, T]$. Since e.g. $\eta_{h t t}=\hat{f}\left(u_{h t}\right)+F\left(\eta_{h t}, u_{h}\right)+F\left(\eta_{h}, u_{h t}\right)$ etc., similar estimates applied recursively yield bounds of the form

$$
\max _{0 \leq t \leq T}\left(\left\|\partial_{t}^{j} \eta_{h}\right\|_{2}+\left\|\partial_{t}^{j} u_{h}\right\|_{1}\right) \leq C_{j}, \quad j=0,1,2, \ldots,
$$

where $C_{j}$ are constants independent of $h$. (A similar result holds in the $W_{\infty}^{1} \times L^{\infty}$ norm.) As is well-known, (16) implies that the o.d.e. system (6) is not stiff and, consequently, may be discretized in time by explicit time-stepping methods in an unconditionally stable manner.

\subsection{Time-stepping with an explicit Runge-Kutta method}

We shall discretize the o.d.e. ivp (6) using the classical, explicit, four-stage Runge-Kutta (RK) method of order four. We shall use a uniform time step $k$, assuming that for a positive integer $N$, $k=T / N$, and putting $t^{n}=n k, n=0,1, \ldots$ Given an o.d.e. system $y^{\prime}=\Phi(t, y)$, one step of this RK scheme (with $y^{n}$ approximating $y\left(t^{n}\right)$ ) is of the form

$$
\begin{aligned}
& y^{n, 1}=y^{n}, t^{n, 1}=t^{n} \\
& \text { for } i=2,3,4 \text { : } \\
& \qquad y^{n, i}=y^{n}+k a_{i} \Phi\left(t^{n, i-1}, y^{n, i-1}\right), t^{n, i}=t^{n}+\tau_{i} k \\
& y^{n+1}=y^{n}+k \sum_{j=1}^{4} b_{j} \Phi\left(t^{n, j}, y^{n, j}\right),
\end{aligned}
$$

where $a_{2}=a_{3}=\frac{1}{2}, a_{4}=1, \tau_{2}=\tau_{3}=\frac{1}{2}, \tau_{4}=1, b_{1}=b_{4}=\frac{1}{6}, b_{2}=b_{3}=\frac{1}{3}$.

Applying this scheme to the ivp (4)-(5), and denoting by $H^{n}, U^{n}$, respectively, the fully discrete approximation in $S_{h}^{0}$ of $\eta\left(\cdot, t^{n}\right), u\left(\cdot, t^{n}\right)$, respectively, we are led to the following time-stepping procedure: We seek functions in $S_{h}^{0}$ denoted by $H^{n}, U^{n}, 0 \leq n \leq N, H^{n, i}, U^{n, i}, 0 \leq n \leq N-1$, $i=1,2,3,4$, such that

$$
\begin{aligned}
& H^{0}=R_{h} \eta_{0}, U^{0}=R_{h} u_{0} \\
& \text { for } n=0,1,2, \ldots, N-1: \\
& \qquad \begin{array}{l}
H^{n, 1}=H^{n}, U^{n, 1}=U^{n} \\
\text { for } i=2,3,4: \\
\quad a_{D}\left(H^{n, i}-H^{n}, \chi\right)=-k a_{i}\left(U_{x}^{n, i-1}+\left(H^{n, i-1} U^{n, i-1}\right)_{x}, \chi\right), \forall \chi \in S_{h}^{0} \\
\quad a_{D}\left(U^{n, i}-U^{n}, \varphi\right)=-k a_{i}\left(\left(H_{x}^{n, i-1}+U^{n, i-1} U_{x}^{n, i-1}, \varphi\right)-c\left(H_{x x}^{n, i-1}, \varphi^{\prime}\right)\right), \forall \varphi \in S_{h}^{0} \\
\quad a_{D}\left(H^{n+1}-H^{n}, \chi\right)=-k \sum_{j=1}^{4} b_{j}\left(U_{x}^{n, j}+\left(H^{n, j} U^{n, j}\right)_{x}, \chi\right), \forall \chi \in S_{h}^{0} \\
\quad a_{D}\left(U^{n+1}-U^{n}, \varphi\right)=-k \sum_{j=1}^{4} b_{j}\left(\left(H_{x}^{n, j}+U^{n, j} U_{x}^{n, j}, \varphi\right)-c\left(H_{x x}^{n, j}, \varphi^{\prime}\right)\right), \forall \varphi \in S_{h}^{0}
\end{array}
\end{aligned}
$$

Hence, given a basis $\left\{\varphi_{i}\right\}$ of $S_{h}^{0}$, the implementation of this scheme requires solving eight linear systems with the same, time-independent matrix $a_{D}\left(\varphi_{i}, \varphi_{j}\right)$. We have the following, optimal-order in $t$, error estimate:

Proposition 10 Let $\mu \geq 1, r \geq \mu+2$ and $(\eta, u)$ be the solution of $(D)$, and suppose that $(\eta, u) \in$ $C\left(0, T ; H^{r} \cap H_{0}^{1}\right) \times C\left(0, T ; H^{r-1} \cap H_{0}^{1}\right)$ for some $0<T<\infty$. Let $H^{n}, U^{n}, 0 \leq n \leq N$, be the solution of (17). Then, for $h$ and $k$ sufficiently small, there exists a constant $C$ independent of $h$ such that 


$$
\max _{0 \leq n \leq N}\left(\left\|H^{n}-\eta\left(t^{n}\right)\right\|_{2}+\left\|U^{n}-u\left(t^{n}\right)\right\|_{1} \leq C\left(k^{4}+h^{r-2}\right) .\right.
$$

PROOF. Let $\eta_{h}, u_{h}$ be the semidiscrete approximations defined by (6) and put for $n=0, \ldots, N-1$, $V^{n, 1}=\eta_{h}^{n}:=\eta_{h}\left(t^{n}\right)$ and $W^{n, 1}=u_{h}^{n}:=u_{h}\left(t^{n}\right)$. Also, let $V^{n, j}, W^{n, j} \in S_{h}^{0}$ for $j=2,3,4$ be defined as $V^{n, j}:=\eta_{h}^{n}+k a_{j} f\left(V^{n, j-1}, W^{n, j-1}\right), W^{n, j}:=u_{h}^{n}+k a_{j} g\left(V^{n, j-1}, W^{n, j-1}\right)$. Hence, the local temporal errors of the RK scheme $\delta_{1}^{n}, \delta_{2}^{n} \in S_{h}^{0}$ are given by

$$
\begin{aligned}
& \delta_{1}^{n}:=\eta_{h}^{n+1}-\eta_{h}^{n}-k \sum_{j=1}^{4} b_{j} f\left(V^{n, j}, W^{n, j}\right), \\
& \delta_{2}^{n}:=u_{h}^{n+1}-u_{h}^{n}-k \sum_{j=1}^{4} b_{j} g\left(V^{n, j}, W^{n, j}\right) .
\end{aligned}
$$

We shall show that under our hypotheses

$$
\max _{0 \leq n \leq N-1}\left(\left\|\delta_{1}^{n}\right\|_{2}+\left\|\delta_{2}^{n}\right\|_{1}\right) \leq C k^{5} .
$$

This will be done by explicitly computing the intermediate stages $V^{n, j}, W^{n, j}$ and the function values $f\left(V^{n, j}, W^{n, j}\right), g\left(V^{n, j}, W^{n, j}\right)$ in terms of the temporal derivatives of $u_{h}$ and $\eta_{h}$ at $t=t^{n}$. First, using the definitions of $f$ and $g$ we obtain

$$
\begin{aligned}
& V^{n, 2}=\eta_{h}^{n}+\frac{k}{2} \eta_{h t}^{n}, W^{n, 2}=u_{h}^{n}+\frac{k}{2} u_{h t}^{n}, \\
& f\left(V^{n, 2}, W^{n, 2}\right)=\eta_{h t}^{n}+\frac{k}{2} \eta_{h t t}^{n}+\frac{k^{2}}{4} \alpha^{n}, g\left(V^{n, 2}, W^{n, 2}\right)=u_{h t}^{n}+\frac{k}{2} u_{h t t}^{n}+\frac{k^{2}}{8} \beta^{n},
\end{aligned}
$$

where $\alpha^{n}=\alpha\left(t^{n}\right), \alpha(t):=F\left(\eta_{h_{t}}(t), u_{h t}(t)\right), \beta^{n}=\beta\left(t^{n}\right), \beta(t):=F\left(u_{h t}(t), u_{h t}(t)\right)$. In addition, we find after long computations that

$$
\begin{gathered}
V^{n, 3}=\sum_{j=0}^{2}\left(\frac{k}{2}\right)^{j} \partial_{t}^{j} \eta_{h}^{n}+\frac{k^{3}}{8} \alpha^{n}, W^{n, 3}=\sum_{j=0}^{2}\left(\frac{k}{2}\right)^{j} \partial_{t}^{j} u_{h}^{n}+\frac{k^{3}}{16} \beta^{n}, \\
f\left(V^{n, 3}, W^{n, 3}\right)=\eta_{h t}^{n}+\frac{k}{2} \eta_{h t t}^{n}+\frac{k^{2}}{4} \eta_{h t t t}^{n}-\frac{k^{2}}{4} \alpha^{n}+\frac{k^{3}}{8}\left[F\left(\alpha^{n}, u_{h}^{n}\right)+\frac{1}{2} f\left(\eta_{h}^{n}, \beta^{n}\right)+\alpha_{t}^{n}\right]+\frac{k^{4}}{16} \gamma_{1}^{n}, \\
g\left(V^{n, 3}, W^{n, 3}\right)=u_{h t}^{n}+\frac{k}{2} u_{h t t}^{n}+\frac{k^{2}}{4} u_{h t t t}^{n}-\frac{k^{2}}{8} \beta^{n}+\frac{k^{3}}{8}\left[\hat{g}\left(\alpha^{n}\right)+\frac{1}{2} F\left(u_{h}^{n}, \beta^{n}\right)+\frac{1}{2} \beta_{t}^{n}\right]+\frac{k^{4}}{32} \gamma_{2}^{n},
\end{gathered}
$$

where $\gamma_{1}^{n}:=F\left(\alpha^{n}, u_{h t}^{n}+\frac{k}{2} u_{h t t}^{n}+\frac{k^{2}}{8} \beta^{n}\right)+\frac{1}{2} F\left(\eta_{h t}^{n}+\frac{k}{2} \eta_{h t t}^{n}, \beta^{n}\right)+F\left(\eta_{h t t}^{n}, u_{h t t}^{n}\right), \gamma_{2}^{n}:=F\left(u_{h t}^{n}+\frac{k}{2} u_{h t t}^{n}+\right.$ $\left.\frac{k^{2}}{16} \beta^{n}, \beta^{n}\right)+F\left(u_{h t t}^{n}, u_{h t t}^{n}\right)$. Finally, we obtain

$V^{n, 4}=\eta_{h}^{n}+k \eta_{h t}^{n}+\frac{k^{2}}{2} \eta_{h t t}^{n}+\frac{k^{3}}{4} \eta_{h t t t}^{n}-\frac{k^{3}}{4} \alpha^{n}+\frac{k^{4}}{8} \hat{\gamma}_{1}^{n}, \quad W^{n, 4}=u_{h}^{n}+k u_{h t}^{n}+\frac{k^{2}}{2} u_{h t t}^{n}+\frac{k^{3}}{4} u_{h t t t}^{n}-\frac{k^{3}}{8} \beta^{n}+\frac{k^{4}}{8} \hat{\gamma}_{2}^{n}$,

where

$$
\begin{aligned}
\hat{\gamma}_{1}^{n} & :=F\left(\alpha^{n}, u_{h}^{n}\right)+\frac{1}{2} f\left(\eta_{h}^{n}, \beta^{n}\right)+\alpha_{t}^{n}+\frac{k}{2} \gamma_{1}^{n}, \\
\hat{\gamma}_{2}^{n} & :=\hat{g}\left(\alpha^{n}\right)+\frac{1}{2} F\left(u_{h}^{n}, \beta^{n}\right)+\frac{1}{2} \beta_{t}^{n}+\frac{k}{4} \gamma_{2}^{n} .
\end{aligned}
$$

Hence,

$$
\begin{aligned}
& f\left(V^{n, 4}, W^{n, 4}\right)=\eta_{h t}^{n}+k \eta_{h t t}^{n}+\frac{k^{2}}{2} \eta_{h t t t}^{n}+\frac{k^{3}}{4} \eta_{h t t t t}^{n}-\frac{k^{3}}{4}\left[\alpha_{t}^{n}+F\left(\alpha^{n}, u_{h}^{n}\right)+\frac{1}{2} f\left(\eta_{h}^{n}, \beta^{n}\right)\right]+\frac{k^{4}}{8} \gamma_{3}^{n}, \\
& g\left(V^{n, 4}, W^{n, 4}\right)=u_{h t}^{n}+k u_{h t t}^{n}+\frac{k^{2}}{2} u_{h t t t}^{n}+\frac{k^{3}}{4} u_{h t t t t}^{n}-\frac{k^{3}}{4}\left[\hat{g}\left(\alpha^{n}\right)+\frac{1}{2} \beta_{t}^{n}+\frac{1}{2} F\left(u_{h}^{n}, \beta^{n}\right)\right]+\frac{k^{4}}{8} \gamma_{4}^{n},
\end{aligned}
$$

where 


$$
\begin{aligned}
\gamma_{3}^{n}:= & \hat{f}\left(\hat{\gamma}_{2}^{n}\right)+F\left(\hat{\gamma}_{1}^{n}, W^{n, 4}-\frac{k^{4}}{8} \hat{\gamma}_{2}^{n}\right)+F\left(V^{n, 4}, \hat{\gamma}_{2}^{n}\right)+F\left(\eta_{h t}^{n}, 2 u_{h t t t}^{n}-\beta^{n}\right) \\
& +F\left(\eta_{h t t}^{n}, 2 u_{h t t}^{n}+k u_{h t t t}^{n}-\frac{k}{2} \beta^{n}\right)+F\left(\eta_{h t t t}^{n}-\alpha^{n}, 2 u_{h t}^{n}+k u_{h t t}^{n}+\frac{k^{2}}{2} u_{h t t t}^{n}-\frac{k^{2}}{4} \beta^{n}\right), \\
\gamma_{4}^{n}:= & \hat{g}\left(\hat{\gamma}_{1}^{n}\right)+F\left(2 u_{h t}^{n}+k u_{h t t}^{n}+\frac{k^{2}}{4} u_{h t t t}^{n}-\frac{k^{2}}{8} \beta^{n}, u_{h t t t}^{n}-\frac{1}{2} \beta^{n}\right)+F\left(u_{h t t}^{n}, u_{h t t}^{n}\right)+F\left(W^{n, 4}-\frac{k^{4}}{16} \hat{\gamma}_{2}^{n}, \hat{\gamma}_{2}^{n}\right) .
\end{aligned}
$$

Hence, as expected (and to our relief), the correct formal order of RK method emerges. Indeed, substituting (21)-(23) into (19) gives

$$
\delta_{1}^{n}=\eta_{h}^{n+1}-\sum_{j=0}^{4} \frac{k^{j}}{j !} \partial_{t}^{j} \eta_{h}^{n}+k^{5} \Gamma_{1}^{n}, \quad \delta_{2}^{n}=u_{h}^{n+1}-\sum_{j=0}^{4} \frac{k^{j}}{j !} \partial_{t}^{j} u_{h}^{n}+k^{5} \Gamma_{2}^{n},
$$

where

$$
\Gamma_{1}^{n}:=-\frac{1}{48}\left(\gamma_{1}^{n}+\gamma_{3}^{n}\right), \quad \Gamma_{2}^{n}:=-\frac{1}{48}\left(\frac{1}{2} \gamma_{2}^{n}+\gamma_{4}^{n}\right) .
$$

As a consequence of (16) and the properties of $f$ and $g$, we see that there exists a constant $C$, independent of $h, k$ and $n$, such that for $0 \leq n \leq N-1,\left\|\alpha^{n}\right\|_{2}+\left\|\beta^{n}\right\|_{1} \leq C,\left\|\gamma_{1}^{n}\right\|_{2}+\left\|\gamma_{2}^{n}\right\|_{1} \leq C$, $\left\|\hat{\gamma}_{1}^{n}\right\|_{2}+\left\|\hat{\gamma}_{2}^{n}\right\|_{1} \leq C,\left\|\gamma_{3}^{n}\right\|_{2}+\left\|\gamma_{4}^{n}\right\|_{1} \leq C,\left\|V^{n, i}\right\|_{2}+\left\|W^{n, i}\right\|_{1} \leq C, 1 \leq i \leq 4$. Therefore, the desired order of accuracy estimate (20) for the local error follows from (24) and (16).

We proceed now with the stability part of the proof. We put $\varepsilon^{n, j}:=V^{n, j}-H^{n, j}, e^{n, j}:=W^{n, j}-$ $U^{n, j}, j=1,2,3,4$, and note that $\varepsilon^{n}:=\varepsilon^{n, 1}=\eta_{h}^{n}-H^{n}, e^{n}:=e^{n, 1}=u_{h}^{n}-U^{n}$. Using the definitions of $f$ and $g$, we obtain, for $0 \leq n \leq N-1, j=2,3,4$,

$$
\begin{aligned}
\varepsilon^{n, j} & =\varepsilon^{n}+k a_{j}\left[f\left(V^{n, j}, e^{n, j}\right)+F\left(\varepsilon^{n, j-1}, W^{n, j-1}\right)-F\left(\varepsilon^{n, j-1}, e^{n, j-1}\right)\right], \\
e^{n, j} & =e^{n}+k a_{j}\left[\hat{g}\left(\varepsilon^{n, j-1}\right)+F\left(W^{n, j-1}, e^{n, j-1}\right)-\frac{1}{2} F\left(e^{n, j-1}, e^{n, j-1}\right)\right] .
\end{aligned}
$$

Suppose now that $\max _{t \in[0, T], 0 \leq i \leq 5}\left(\left\|\partial_{t}^{i} \eta(t)\right\|_{2}+\left\|\partial_{t}^{i} u(t)\right\|_{1}\right) \leq \hat{M}$, where $\hat{M}$ is large enough so that, cf. (16), $\max _{t \in[0, T], 0 \leq i \leq 5}\left(\left\|\partial_{t}^{i} \eta_{h}(t)\right\|_{2}+\left\|\partial_{t}^{i} u_{h}(t)\right\|_{1}\right) \leq 2 \hat{M}$. Let $n^{*}<N$ be the largest integer for which $\left\|H^{n}\right\|_{2}+\left\|U^{n}\right\|_{1} \leq 3 \hat{M}, 0 \leq n \leq n^{*}$. Then, for $0 \leq n \leq n^{*},\left\|\varepsilon^{n}\right\|_{2}+\left\|e^{n}\right\|_{1} \leq 5 \hat{M}$ and $\left\|\varepsilon^{n, j}\right\|_{2}+\left\|e^{n, j}\right\|_{1} \leq\left\|\varepsilon^{n}\right\|_{2}+\left\|e^{n}\right\|_{1}+C k\left(\left\|\varepsilon^{n, j-1}\right\|_{2}+\left\|e^{n, j-1}\right\|_{1}\right), j=2,3,4$, where $C=C(\hat{M})$. Since now

$$
\begin{aligned}
& \varepsilon^{n+1}=\varepsilon^{n}+k \sum_{j=1}^{4} b_{j}\left[f\left(V^{n, j}, W^{n, j}\right)-f\left(H^{n, j}, U^{n, j}\right)\right]+\delta_{1}^{n}, \\
& e^{n+1}=e^{n}+k \sum_{j=1}^{4} b_{j}\left[g\left(V^{n, j}, W^{n, j}\right)-g\left(H^{n, j}, U^{n, j}\right)\right]+\delta_{2}^{n},
\end{aligned}
$$

it follows (with $\left.A^{n}:=\left\|\varepsilon^{n}\right\|_{2}+\left\|e^{n}\right\|_{1}, A^{n, j}:=\left\|\varepsilon^{n, j}\right\|_{2}+\left\|e^{n, j}\right\|_{1}\right)$ that $A^{n+1} \leq(1+k C) A^{n}+C k^{5}$, $A^{0}=0$. Hence $A^{n+1} \leq C k^{4}$, and for $k$ sufficiently small we have $\left\|H^{n+1}\right\|_{2}+\left\|U^{n+1}\right\|_{1} \leq A^{n}+2 \hat{M} \leq$ $C k^{4}+2 \hat{M}<3 \hat{M}$, contradicting the maximal property of $n^{*}$. We conclude that $\left\|H^{n}\right\|_{2}+\left\|U^{n}\right\|_{1} \leq C$ for $0 \leq n \leq N$, whence $\max _{0 \leq n \leq N}\left(\left\|\varepsilon^{n}\right\|_{2}+\left\|e^{n}\right\|_{1}\right)=\max _{0 \leq n \leq N}\left(\left\|\eta_{h}^{n}-H^{n}\right\|_{2}+\left\|u_{h}^{n}-U^{n}\right\|_{1}\right) \leq C k^{4}$. The estimate (18) now follows from (7).

Using similar estimates and Proposition 6 one may also prove

Proposition 11 Let $\mu \geq 1, r \geq \mu+1$ and $(\eta, u)$, the solution of $(D)$, belong to the space $C\left(0, T ; C_{0}^{r}\right) \times$ $C\left(0, T ; C_{0}^{r-1}\right)$ for some $0<T<\infty$. Let $H^{n}, U^{n}, 0 \leq n \leq N$, be the solution of (17). Then for $h$ and $k$ sufficiently small, there exists a constant $C$ independent of $h$ and $k$ such that

$$
\max _{0 \leq n \leq N}\left(\left\|H^{n}-\eta\left(t^{n}\right)\right\|_{W_{\infty}^{1}}+\left\|U^{n}-u\left(t^{n}\right)\right\|_{L^{\infty}}\right) \leq C\left(k^{4}+h^{r-1}\right) .
$$


For the BBM-BBM system one may easily prove optimal-order $O\left(k^{4}+h^{r}\right) L^{2}$-estimates for both components of the error.

\subsection{Discretization of the ibvp with reflection boundary conditions}

We consider now the ibvp, that we will denote by $(\mathrm{R})$ and which is obtained by $(\mathrm{D})$ if we replace the Dirichlet boundary conditions by $\eta_{x}( \pm L, t)=0, u( \pm L, t)=0, t \geq 0$. Let again $\mu \geq 1$ and $r \geq \mu+2$. The standard Galerkin semidiscretization of this ibvp in $S_{h} \times S_{h}^{0}$ is defined as follows. We seek $\eta_{h}:[0, T] \rightarrow S_{h}$ and $u_{h}:[0, T] \rightarrow S_{h}^{0}$ satisfying for $0 \leq t \leq T$

$$
\begin{aligned}
& a_{N}\left(\eta_{h_{t}}, \chi\right)+\left(\left(u_{h}+\eta_{h} u_{h}\right)_{x}, \chi\right)=0, \forall \chi \in S_{h}, \\
& a_{D}\left(u_{h t}, \chi\right)+\left(\eta_{h_{x}}, \psi\right)-c\left(\eta_{h_{x x}}, \psi^{\prime}\right)+\left(u_{h} u_{h x}, \psi\right)=0, \forall \psi \in S_{h}^{0},
\end{aligned}
$$

with initial conditions

$$
\eta_{h}(0)=R_{h} \eta_{0}, \quad u_{h}(0)=R_{h}^{0} u_{0} .
$$

In addition to the mappings $\hat{f}, \hat{g}, F, g$ defined in Paragraph 2.2, we shall also need the mappings $\tilde{f}: L^{2} \rightarrow S_{h}$, defined by $a_{N}(\tilde{f}(w), \phi)=\left(w, \phi^{\prime}\right), \forall \phi \in S_{h}, \tilde{F}: H^{1} \times H^{1} \rightarrow S_{h}$, defined by $\tilde{F}(v, w)=$ $\tilde{f}(v w), f_{R}: H^{1} \times H^{1} \rightarrow S_{h}$, defined by $f_{R}(v, w)=\tilde{f}(w)+\tilde{F}(v, w)$. Then, we may write (25)-(26) as

$$
\begin{aligned}
& \eta_{h_{t}}=f_{R}\left(\eta_{h}, u_{h}\right), \quad 0 \leq t \leq T, \\
& u_{h t}=g\left(\eta_{h}, u_{h}\right), \\
& \eta_{h}(0)=R_{h} \eta_{0}, u_{h}(0)=R_{h}^{0} u_{0} .
\end{aligned}
$$

Following the general scheme of the proofs in Lemma 4 , it may be shown that $f_{R}$ satisfies analogous properties to (i), (ii) and (iii) of Lemma 7. Hence, it may be checked that the following analogs of Propositions 5 and 6 hold for (27) as well. The proofs are omitted.

Proposition 12 Suppose that the classical solution $(\eta, u)$ of $(R)$ lies in $C\left(0, T ; H^{r}\right) \times C\left(0, T ; H^{r-1} \cap\right.$ $\left.H_{0}^{1}\right)$. Then, if $h$ is sufficiently small, the semidiscrete problem (27) has a unique solution $\left(\eta_{h}, u_{h}\right)$ on $[0, T]$, which satisfies, for some constant $C$ independent of $h$,

$$
\left\|\eta(t)-\eta_{h}(t)\right\|_{2}+\left\|u(t)-u_{h}(t)\right\|_{1} \leq C h^{r-2}, \quad 0 \leq t \leq T .
$$

Proposition 13 Suppose that the classical solution $(\eta, u)$ of $(R)$ lies in $C\left(0, T ; C^{r}\right) \times C\left(0, T ; C_{0}^{r-1}\right)$. Then, for $h$ sufficiently small, there exists a constant $C$, independent of $h$, such that

$$
\left\|\eta(t)-\eta_{h}(t)\right\|_{W_{\infty}^{1}}+\left\|u(t)-u_{h}(t)\right\|_{L^{\infty}} \leq C h^{r-1} .
$$

For the analogous to (17) temporal discretization of (25)-(26), denoting again by $H^{n}, U^{n}$ the fully discrete approximations of $\eta\left(t^{n}\right), u\left(t^{n}\right)$, we may prove the analogs of Propositions 10 and 11 , which we combine into the following

Proposition 14 Suppose that the classical solution $(\eta, u)$ of $(R)$ has the regularity properties stated in Proposition 12 and 13, respectively. Then, for $k$ and $h$ sufficiently small, we have, respectively

$$
\begin{aligned}
& \max _{0 \leq n \leq N}\left(\left\|H^{n}-\eta\left(t^{n}\right)\right\|_{2}+\left\|U^{n}-u\left(t^{n}\right)\right\|_{1}\right) \leq C\left(k^{4}+h^{r-2}\right), \\
& \max _{0 \leq n \leq N}\left(\left\|H^{n}-\eta\left(t^{n}\right)\right\|_{W_{\infty}^{1}}+\left\|U^{n}-u\left(t^{n}\right)\right\|_{L^{\infty}}\right) \leq C\left(k^{4}+h^{r-1}\right) .
\end{aligned}
$$

In the case of the BBM-BBM system one may prove analogous estimates of optimal spatial order $O\left(h^{r}\right)$ in $L^{2}$. 


\subsection{Discretization of the periodic initial-value problem}

We finally discretize the periodic initial-value problem (ipvp) (P) for (1) with periodic boundary conditions at $x= \pm L$, where $\eta_{0}, u_{0}$ are given $2 L$-periodic functions. For this purpose we shall use the space of smooth periodic splines on a uniform mesh on $\bar{I}=[-L, L]$ for the spatial discretization. It is well-known, [35], that optimal-order $L^{2}$ error estimates are valid in this spline space for the semidiscretization of ipvp's of linear pde's with variable-coefficient, $L^{2}$-semibounded spatial operators of any (odd or even) order. This error analysis has been extended in the case of the KdV equation and its variants, cf. e.g. [5], [19], [9], and may also be used for Boussinesq systems. In what follows, we shall just establish notation and state some relevant error estimates, whose proofs may be found in [1].

Let $J$ be a positive integer and put $h=2 L / J$. Consider the uniform partition of $\bar{I}$ given by $x_{j}=-L+j h, j=0, \ldots, J$. For integer $r \geq 3$ we consider the associated $J$-dimensional space of smooth periodic splines on $\bar{I}$

$$
S_{h}=\left\{\phi \in C_{p}^{r-2}:\left.\phi\right|_{\left[x_{j}, x_{j+1}\right]} \in \mathbb{P}_{r-1}, 0 \leq j \leq J-1\right\} .
$$

It is well-known that given $w$ in the Sobolev space $H_{p}^{s}$ (which consists of the $2 L$-periodic functions in $H^{s}$ ) there exists a $\chi \in S_{h}$ such that $\sum_{j=0}^{s-1} h^{j}\|w-\chi\|_{j} \leq C h^{s}\|w\|_{s}, \quad 1 \leq s \leq r$, for some constant $C$ independent of $h$ and $w$. In addition, cf. [35] and the references quoted therein, one may construct a basis $\left\{\phi_{j}\right\}_{j=1}^{J}$ of $S_{h}$, so that, for $w \in H_{p}^{r}$ the associated quasiinterpolant $Q_{h} w=\sum_{j=1}^{J} w\left(x_{j}\right) \phi_{j}(x)$ satisfies $\left\|w-Q_{h} w\right\| \leq C h^{r}\left\|w^{(r)}\right\|$.

The semidiscrete approximation $\left(\eta_{h}, u_{h}\right):[0, T] \rightarrow S_{h} \times S_{h}$ of the solution $(\eta, u)$ of $(\mathrm{P})$ is defined by

$$
\begin{aligned}
& a_{p}\left(\eta_{h t}, \chi\right)+\left(\left(u_{h}+\eta_{h} u_{h}\right)_{x}, \chi\right)=0, \forall \chi \in S_{h}, \\
& a_{p}\left(u_{h t}, \phi\right)+\left(\eta_{h_{x}}+u_{h} u_{h x}, \phi\right)-c\left(\eta_{h x x}, \phi^{\prime}\right)=0, \quad \forall \phi \in S_{h}, \\
& \eta_{h}(0)=Q_{h} \eta_{0}, \quad u_{h}(0)=Q_{h} u_{0},
\end{aligned}
$$

where $a_{p}(v, w):=(v, w)+b\left(v^{\prime}, w^{\prime}\right)$ for $v, w \in H_{p}^{1}$. The first step in the proof of the $L^{2}$-error estimates is to establish optimal-rate $L^{2}$ error bounds for a suitable local error of (28). This may be accomplished by using some properties of the quasiinterpolant proved in [35], cf. [5], [19], [1].

Lemma 15 Suppose that the solution $(\eta, u)$ of $(P)$ is sufficiently smooth on $[0, T]$. Let $H:=Q_{h} \eta$, $U:=Q_{h} u$, and $\psi, \zeta \in C\left(0, T ; S_{h}\right)$ be such that

$$
\begin{aligned}
& a_{p}\left(H_{t}, \chi\right)+\left((U+H U)_{x}, \chi\right)=(\psi, \chi), \forall \chi \in S_{h}, \\
& a_{p}\left(U_{t}, \phi\right)+\left(H_{x}+U U_{x}, \phi\right)-c\left(H_{x x}, \phi^{\prime}\right)=(\zeta, \phi), \quad \forall \phi \in S_{h} .
\end{aligned}
$$

Then, for some constant $C$ independent of $h$ we have

$$
\max _{0 \leq t \leq T}(\|\psi(t)\|+\|\zeta(t)\|) \leq C h^{r} .
$$

With the aid of this lemma, and using a stability argument similar to the one in the proof of Proposition 5, one may prove the superaccuracy result that $\left\|Q_{h} \eta-\eta_{h}\right\|_{2}+\left\|Q_{h} u-u_{h}\right\|_{1} \leq C h^{r}$, on $[0, T]$, cf. [1], from which there follows

Proposition 16 Let the solution $(\eta, u)$ of $(P)$ be sufficiently smooth in $[0, T]$. Then, for $h$ sufficiently small, the solution $\left(\eta_{h}, u_{h}\right)$ of (28) exists in $[0, T]$ and satisfies, for some constant $C$ independent of $h$, 


$$
\max _{0 \leq t \leq T}\left(\left\|\eta(t)-\eta_{h}(t)\right\|+\left\|u(t)-u_{h}(t)\right\|\right) \leq C h^{r} .
$$

Discretizing the o.d.e. system (28) in time in the usual way by the classical, fourth-order explicit RK method of Paragraph 2.3 and denoting by $H_{h}^{n}, U_{h}^{n}, 0 \leq n \leq N$, the fully discrete approximations of $\eta\left(t^{n}\right), u\left(t^{n}\right)$, we may prove now, cf. [1], that

$$
\max _{0 \leq t \leq T}\left(\left\|H_{h}^{n}-\eta\left(t^{n}\right)\right\|+\left\|U_{h}^{n}-u\left(t^{n}\right)\right\|\right) \leq C\left(k^{4}+h^{r}\right) .
$$

Optimal-order in space and time $L^{2}$-error estimates may be proved in the case of the periodic ivp for the BBM-BBM system, and for other Boussinesq systems. The proofs will appear elsewhere.

\section{Numerical experiments}

In what follows we present a series of numerical experiments aimed at checking the accuracy of the numerical scheme put forth in the previous section and illustrating the behavior of solutions of the Bona-Smith systems in some interesting examples. We used throughout cubic splines (i.e. the space $S_{h}$ with $\mu=2, r=4$ in the notation of Paragraph 2.1) on a uniform mesh for the spatial discretization, while the time-stepping was effected by the classical, four-stage, fourth-order RK scheme. As initial conditions throughout were taken the appropriate elliptic projections of the initial data.

\subsection{Numerical study of convergence rates}

We begin by presenting an experimental study of the rates of the spatial convergence of the numerical scheme in various norms for the three types of boundary conditions considered. In each case the spatial interval was $[0,1]$. We took $h=1 / N$ and $h=k$ and integrated up to $T=2$. In each case we integrated with a version of the code that was supplied with a right-hand side nonhomogeneous term, computed from the assumed exact solution, and we computed the $L^{2}, H^{1}$, $H^{2}, L^{\infty}$ and $W_{\infty}^{1}$ norms of the errors for several $N$ and also the resulting convergence rates. (We checked that the $O\left(k^{4}\right)$ temporal error was always sufficiently small compared to the spatial error.) The maximum values needed to compute the $L^{\infty}$ and $W_{\infty}^{1}$ norms were taken over all quadrature points in $[0,1]$ used in the finite element quadratures. (We employed the 5 -point Gauss rule in each spatial mesh interval.)

(i) Dirichlet boundary conditions

As exact solution we took the pair of functions $\eta(x, t)=x(x-1) e^{2 t} \cos \pi x, u(x, t)=2 e^{x t} \sin \pi x$ that satisfy homogeneous Dirichlet boundary conditions at $x=0$ and $x=1$. The values of the errors and associated convergence rates are shown in Table 1 in the case of the system $\theta^{2}=9 / 11$. The observed spatial rates of the $\eta$-errors were practically equal to $3,3,2,3$, and 3 in the $L^{2}, H^{1}$, $H^{2}, L^{\infty}$ and $W_{\infty}^{1}$ norms, respectively. This indicates that the optimal-order bounds obtained in Proposition 5 for the $H^{2}$ norm and in Proposition 6 for the $W_{\infty}^{1}$ norm of the $\eta$-error are sharp. It also suggests that the standard Galerkin method, in the case of Dirichlet boundary conditions, yields suboptimal rates of convergence for $\eta$ in $L^{2}$ and $L^{\infty}$ and optimal rates in $H^{1}$ and $W_{\infty}^{1}$. The analogous rates for the $u$-errors were approximately equal to $3,2.5,1.5,3$, and 2 , in the $L^{2}, H^{1}$, $H^{2}, L^{\infty}$, and $W_{\infty}^{1}$ norms, respectively. This indicates that the order 2 for $\left\|u-u_{h}\right\|_{1}$ in the bound in (7) is pessimistic by $1 / 2$, while the order 3 for $\left\|u-u_{h}\right\|_{L^{\infty}}$ in (15) is again sharp. It also suggests that the standard Galerkin method, in the case of Dirichlet boundary conditions, yields suboptimal rates of convergence for $u$ in all norms. In the case of the easier to integrate $\theta^{2}=2 / 3$ (BBM-BBM) system, where only $\partial_{x x t}$ derivatives are present, all rates are as expected, optimal (i.e. equal to 4 , $3,2,4$ and 3 for the errors in the $L^{2}, H^{1}, H^{2}, L^{\infty}$, and $W_{\infty}^{1}$, respectively for both $\eta$ and $u$.) 
Table 1

Errors and spatial convergence rates at $T=2$. Homogeneous Dirichlet boundary conditions. System with $\theta^{2}=9 / 11$.

\begin{tabular}{lllll|llll}
\hline $\mathrm{N}$ & $\eta$-error & rate & $u$-error & rate & $\eta$-error & rate & $u$-error & rate \\
\hline \multicolumn{7}{c}{$L^{2}$-errors } & & \multicolumn{3}{c}{$H^{1}$-errors } \\
20 & $0.1165(-2)$ & 3.065 & $0.1696(-2)$ & 3.087 & $0.6786(-2)$ & 3.030 & $0.2778(-1)$ & 2.562 \\
40 & $0.1439(-3)$ & 3.016 & $0.2086(-3)$ & 3.023 & $0.8450(-3)$ & 3.006 & $0.4913(-2)$ & 2.499 \\
80 & $0.1795(-4)$ & 3.004 & $0.2596(-4)$ & 3.006 & $0.1057(-3)$ & 2.999 & $0.8740(-3)$ & 2.491 \\
160 & $0.2243(-5)$ & 3.001 & $0.3240(-5)$ & 3.002 & $0.1322(-4)$ & 2.999 & $0.1552(-3)$ & 2.493 \\
320 & $0.2803(-6)$ & 3.000 & $0.4048(-6)$ & 3.001 & $0.1653(-5)$ & 2.999 & $0.2752(-4)$ & 2.496 \\
\hline & & $H^{2}$-errors & & & & $L^{\infty}$-errors & \\
\hline 10 & 2.5660 & & 6.3047 & & $0.1568(-1)$ & & $0.2452(-1)$ & \\
20 & 0.6232 & 2.042 & 2.1878 & 1.527 & $0.1859(-2)$ & 3.077 & $0.2708(-2)$ & 3.179 \\
40 & 0.1547 & 2.010 & 0.7805 & 1.487 & $0.2284(-3)$ & 3.025 & $0.3215(-3)$ & 3.075 \\
80 & $0.3859(-1)$ & 2.003 & 0.2788 & 1.485 & $0.2840(-4)$ & 3.008 & $0.3927(-4)$ & 3.033 \\
160 & $0.9642(-2)$ & 2.001 & $0.9924(-1)$ & 1.490 & $0.3542(-5)$ & 3.003 & $0.4856(-5)$ & 3.016 \\
320 & $0.2410(-2)$ & 2.000 & $0.3522(-1)$ & 1.495 & $0.4422(-6)$ & 3.002 & $0.6039(-6)$ & 3.007 \\
\hline
\end{tabular}

\begin{tabular}{lllll}
\hline \multicolumn{5}{c}{$W_{\infty}^{1}$-errors } \\
$\mathrm{N}$ & $\eta$-error & rate & $u$-error & rate \\
\hline 10 & 0.1212 & & 0.9519 & \\
40 & $0.1576(-1)$ & 2.942 & 0.2153 & 2.144 \\
40 & $0.1969(-2)$ & 3.001 & $0.5174(-1)$ & 2.057 \\
80 & $0.2450(-3)$ & 3.007 & $0.1272(-1)$ & 2.024 \\
160 & $0.3064(-4)$ & 2.999 & $0.3157(-2)$ & 2.011 \\
320 & $0.3834(-5)$ & 2.998 & $0.7864(-3)$ & 2.005 \\
\hline
\end{tabular}

(ii) Reflection boundary conditions

In this case the observed convergence rates for the $\eta$ and $u$ errors for all norms for the systems corresponding to $\theta^{2}=9 / 11$ are practically the same with those of Table 1 and are not shown here. Identical remarks with those made in the case of Dirichlet boundary conditions on the optimality and non-optimality of the convergence rates apply, in view e.g. of the results of Propositions 12 and 13. All rates for the BBM-BBM system are again optimal.

(iii) Periodic boundary conditions

As exact solution in this case we took the pair of functions $\eta=e^{t} \sin [2 \pi(x-2 t)], u=2 e^{t / 2} \cos [2 \pi(x-$ $t / 2)$ ]. The observed convergence rates for the system with $\theta^{2}=9 / 11$ are shown in Table 2 . All rates for both components of the error are of optimal order as expected. The analogous experiment with the BBM-BBM system gave the same rates. It is interesting to report that at the nodes $x_{j}=j h$ of 
the uniform spatial discretization we observed superconvergence at a $O\left(h^{4}\right)$ rate of the $W_{\infty}^{1}$ norms of the errors of $\eta$ and $u$. (Of course, superconvergence at the nodes is a well-known phenomenon for periodic initial-value problems for linear p.d.e.'s with constant coefficients, cf. e.g. [35].)

Table 2

Errors and spatial convergence rates at $T=2$. Periodic boundary conditions. System with $\theta^{2}=9 / 11$.

\begin{tabular}{|c|c|c|c|c|c|c|c|c|}
\hline $\mathrm{N}$ & $\eta$-error & rate & $u$-error & rate & $\eta$-error & rate & $u$-error & rate \\
\hline \multicolumn{5}{|c|}{$L^{2}$-errors } & \multicolumn{4}{|c|}{$H^{1}$-errors } \\
\hline 10 & $0.8919(-2)$ & & $0.1162(-1)$ & & $0.8783(-1)$ & & $0.8826(-1)$ & \\
\hline 20 & $0.3657(-3)$ & 4.608 & $0.8027(-3)$ & 3.855 & $0.6832(-2)$ & 3.684 & $0.6838(-2)$ & 3.690 \\
\hline 40 & $0.1841(-4)$ & 4.312 & $0.5184(-4)$ & 3.953 & $0.7580(-3)$ & 3.172 & $0.6370(-3)$ & 3.424 \\
\hline 80 & $0.1038(-5)$ & 4.149 & $0.3273(-5)$ & 3.985 & $0.9223(-4)$ & 3.039 & $0.7064(-4)$ & 3.173 \\
\hline 160 & $0.6180(-7)$ & 4.070 & $0.2052(-6)$ & 3.995 & $0.1146(-4)$ & 3.009 & $0.8519(-5)$ & 3.052 \\
\hline 320 & $0.3775(-8)$ & 4.033 & $0.1284(-7)$ & 4.998 & $0.1430(-5)$ & 3.002 & $0.1055(-5)$ & 3.014 \\
\hline \multicolumn{5}{|c|}{$H^{2}$-errors } & \multicolumn{4}{|c|}{$L^{\infty}$-errors } \\
\hline 10 & 3.2648 & & 2.4343 & & $0.1838(-1)$ & & $0.1965(-1)$ & \\
\hline 20 & 0.7685 & 2.087 & 0.5660 & 2.105 & $0.8098(-3)$ & 4.504 & $0.1202(-2)$ & 4.031 \\
\hline 40 & 0.1902 & 2.014 & 0.1400 & 2.016 & $0.4227(-4)$ & 4.260 & $0.7396(-4)$ & 4.023 \\
\hline 80 & $0.4745(-1)$ & 2.003 & $0.3491(-1)$ & 2.003 & $0.2422(-5)$ & 4.125 & $0.4661(-5)$ & 3.988 \\
\hline 160 & $0.1186(-1)$ & 2.001 & $0.8724(-2)$ & 2.001 & $0.1452(-6)$ & 4.060 & $0.2947(-6)$ & 3.983 \\
\hline \multirow[t]{9}{*}{320} & $0.2964(-2)$ & 2.000 & $0.2181(-2)$ & 2.000 & $0.8896(-8)$ & 4.029 & $0.1852(-7)$ & 3.993 \\
\hline & & \multicolumn{5}{|c|}{$W_{\infty}^{1}$-errors } & & \\
\hline & & $\mathrm{N}$ & $\eta$-error & rate & $u$-error & rate & & \\
\hline & & 10 & 0.1939 & & 0.2044 & & & \\
\hline & & 20 & $0.1631(-1)$ & 3.572 & $0.1732(-1)$ & 3.561 & & \\
\hline & & 40 & $0.1686(-2)$ & 3.274 & $0.1617(-2)$ & 3.421 & & \\
\hline & & 80 & $0.1928(-3)$ & 3.128 & $0.1668(-3)$ & 3.277 & & \\
\hline & & 160 & $0.2317(-4)$ & 3.057 & $0.1865(-4)$ & 3.161 & & \\
\hline & & 320 & $0.2845(-5)$ & 3.026 & $0.2195(-5)$ & 3.087 & & \\
\hline
\end{tabular}

\subsection{Amplitude-speed relationship for solitary waves}

As pointed out in the Introduction, we will use the numerical method previously analyzed and tested experimentally as a tool to study numerically interactions of solitary-wave solutions of the Bona-Smith systems. Some such exact solutions are known in closed form. Following e.g. the procedure in [14] we may find, for each $\theta^{2} \in(7 / 9,1)$ a single solitary-wave solution of the corresponding system, of the form 


$$
\eta_{s}(x, t)=\eta_{0} \operatorname{sech}^{2}\left(\lambda\left(x+x_{0}-c_{s} t\right)\right), \quad u_{s}(x, t)=B \eta_{s}(x, t),
$$

where $x_{0}$ is arbitrary and

$$
\begin{aligned}
& \eta_{0}=\frac{9}{2} \frac{\theta^{2}-7 / 9}{1-\theta^{2}}, \quad c_{s}=\frac{4\left(\theta^{2}-2 / 3\right)}{\sqrt{2\left(1-\theta^{2}\right)\left(\theta^{2}-1 / 3\right)}}, \\
& \lambda=\frac{1}{2} \sqrt{\frac{3\left(\theta^{2}-7 / 9\right)}{\left(\theta^{2}-1 / 3\right)\left(\theta^{2}-2 / 3\right)}}, \quad B=\sqrt{\frac{2\left(1-\theta^{2}\right)}{\theta^{2}-1 / 3}} .
\end{aligned}
$$

As this population of solutions is limited, it is important to be able to construct highly accurate approximations of solitary waves. This may be done by isolating solitary waves in long time simulations, after they separate from the rest of the solution evolving from suitable initial profiles, by 'cleaning' solitary waves by an iterative process, cf. e.g. [6], [18], by solving numerically suitable two-point boundary-value problems for the nonlinear system (3) that solitary waves satisfy, or by some combination of these techniques. A detailed study of the accuracy of the first two methods in the case of the Bona-Smith systems was done in [18] and will not be repeated here. We have checked that the numerical solitary waves used in the numerical experiments to be presented in the sequel are sufficiently accurate in that they have amplitudes that are constant to at least six digits, speeds that remain constant to at least five digits, and propagate shedding dispersive tails of at most $O\left(10^{-10}\right)$ amplitude in the temporal range of experiments.

A natural question that may be asked is how the solitary waves of the Bona-Smith systems compare, theoretically and experimentally, with solitary waves of the Euler equations. As was done in [6], a partial answer may be furnished by comparing pairs $\left(A_{s}, c_{s}\right)$ of the maximum height $A_{s}$ of the $\eta$-solitary wave and its speed $c_{s}$, obtained numerically, and with the expansion, cf. [23], [32]

$$
c_{s}=1+\frac{1}{2} A_{s}-\frac{3}{20}\left(A_{s}\right)^{2}+\frac{3}{56}\left(A_{s}\right)^{3}+\cdots
$$

that provides an approximation of the speed-amplitude relation for solitary waves of the Euler equation for small $A_{s}$, and with the experimental observations of Scott Russell, [30], fitted by the relation

$$
c_{s}=\sqrt{1+A_{s}}
$$

In Figure 1 we compare the graphs of (31) with two, three and four terms, and of (32), with numerically computed solitary-wave data for the Bona-Smith systems when $\theta^{2}=1, \frac{9}{11}$, and $\frac{2}{3}$. The points corresponding to these systems remain close to Scott Russell's prediction for a quite large range of amplitudes (even in the non-physical regime) and stay below the line $c_{s}=1+\frac{A_{s}}{2}$ of the speed-amplitude relationship of the solitary waves of the one-way models (KdV and BBM.)

\subsection{Interaction of solitary waves}

In this paragraph we present the results of some numerical experiments that we performed to study interactions of solitary waves of the Bona-Smith systems. The interactions were of two kinds: Head-on collisions of solitary waves of equal and unequal amplitudes, and overtaking interactions of solitary waves travelling in the same direction. In all cases, the initial solitary waves were constructed by solving numerically a two-point boundary-value problem of the nonlinear system of o.d.e.'s (3), cf. [10], [21]. The initial $\eta$-pulses were nonnegative and centered sufficiently far from each other; their associated initial velocity profiles $(u)$ were of opposite signs for a collision and nonnegative for one-way interactions. 

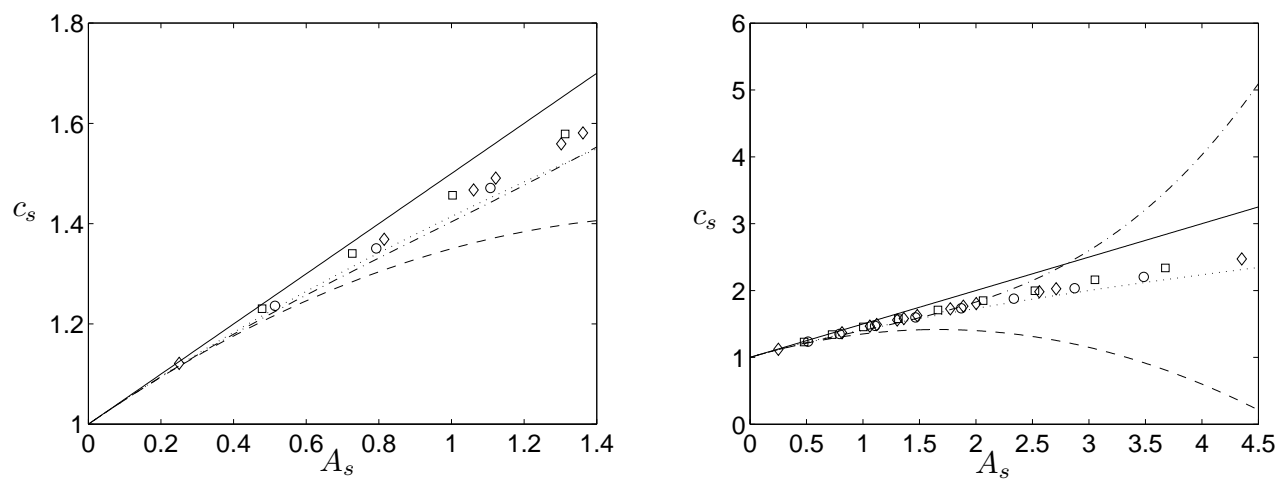

Fig. 1. Speed $c_{s}$ of $\eta$-solitary waves as a function of their amplitude $A_{s}$. '-' 2 terms, '- -' 3 terms, '-.-' 4 terms of (31), ‥ Scott Russell's prediction (32), Bona-Smith systems: $\circ \theta^{2}=2 / 3, \square \theta^{2}=1, \diamond \theta^{2}=9 / 11$.

\subsubsection{Head-on collisions}

A systematic numerical study of head-on collisions of solitary waves of the BBM-BBM system $\left(\theta^{2}=2 / 3\right)$ was performed in [6] by Bona and Chen. Comparisons were also made in [6] of the numerically computed maximum value of the amplitude during the collision and of the accompanying phase shift with theoretical expansions of these quantities formally valid for the Euler equations, cf. e.g. [32], and fairly good agreement was observed. It is not our intention here to repeat this detailed study for other members of the Bona-Smith family of systems. Instead, we would like to point out some similarities and differences in the details of this type of interaction between the $\theta^{2} \neq 2 / 3$ systems and the BBM-BBM. For a recent study of head-on collisions of solitary waves of the Euler equations and review of the relevant literature we refer the reader to [16].

All our head-on collision experiments were performed on $[-150,150]$ using $h=0.1$ and $k=0.01$ and periodic boundary conditions. We first consider the Bona-Smith system with $\theta^{2}=1$. A solitary wave, which at $t=0$ is centered at $x=-50$ and has initial amplitude $A_{1}=0.866190$ and speed $c_{s, 1}=1.4$, travels to the right and collides with a leftward-travelling solitary wave, centered initially at $x=50$ and having initial amplitude $A_{2}=0.412617$ and speed $c_{s, 2}=1.2$. The waves start colliding at about $t=33$, they interact, emerging largely unchanged, and continue travelling in their respective directions. The evolution of the $\eta$-profile of the solution for $0 \leq t \leq 100$ is shown in Figure 2(a), while 2(b) depicts the profile of $\eta(x, t)$ at $t=100$. The collision is inelastic and nonlinear; small-amplitude oscillatory dispersive tails that follow the solitary waves are produced after the interaction. These dispersive tails are shown at $t=100$ in Figure 2(c), a magnification of Figure 2(b), and were studied in some detail in [18]. Figure 2(d) shows the paths of the two waves on the $x, t$ plane around the time of interaction. The solid lines are the actual paths while the dotted lines would represent the paths if there was no interaction. Both waves are slightly delayed after the interaction suffering small phase shifts opposed to their directions of motion. (For example, at $t=80$ the large solitary wave has a phase shift of 0.3194 and the small one of 0.5102 spatial units.)

After the interaction the large solitary wave stabilizes at a slightly smaller amplitude $A_{1}^{\prime}=$ 0.865921 and speed $c_{s, 1}^{\prime}=1.39988$, while the small one emerges with $A_{2}^{\prime}=0.412543, c_{s, 2}^{\prime}=1.19994$. Figure 3 shows the maximum amplitude of the $\eta$ component of the solution as a function of $t, 0 \leq$ $t \leq 100$. The maximum height that the solution reaches during the collision is equal to 1.384417 , which is about $8.3 \%$ larger than the sum of the heights of the two solitary waves before the collision. It is worthwhile to note that after the collision both solitary waves suffer a slight loss of amplitude which seems to be permanent. The temporal transition from the maximum value of the amplitude to the apparent long-time limit (which is of course the eventual amplitude of the emerging larger 


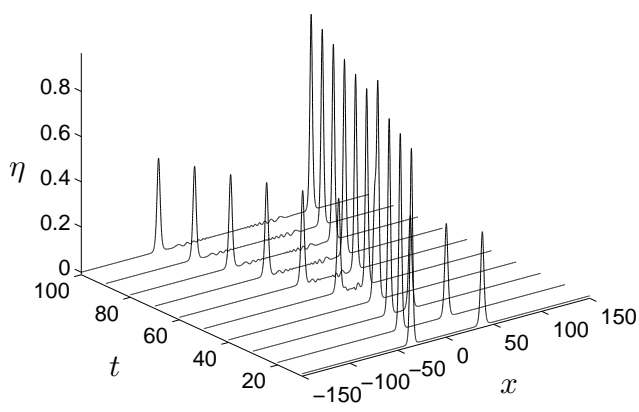

(a)

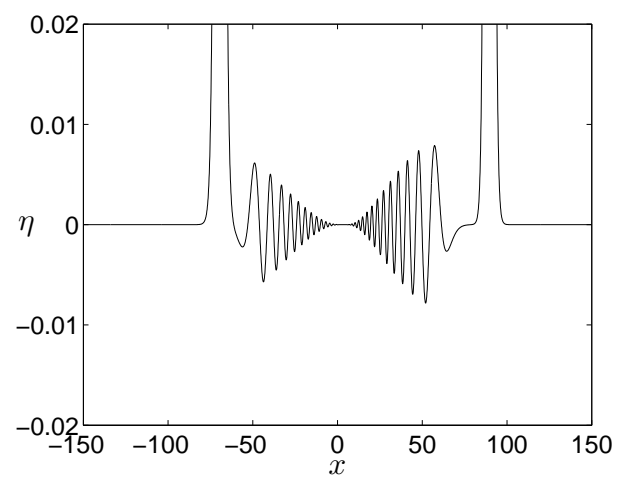

(c)

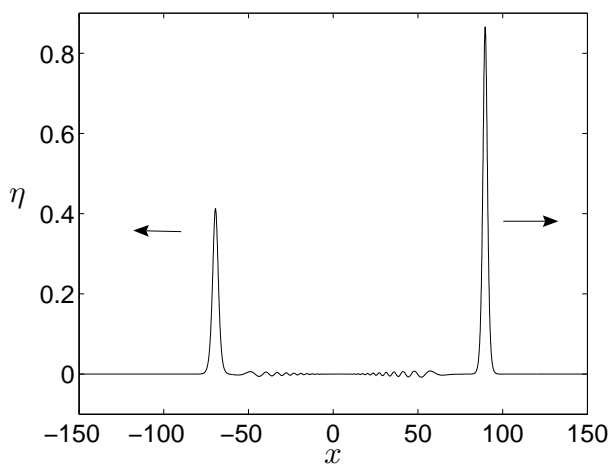

(b)

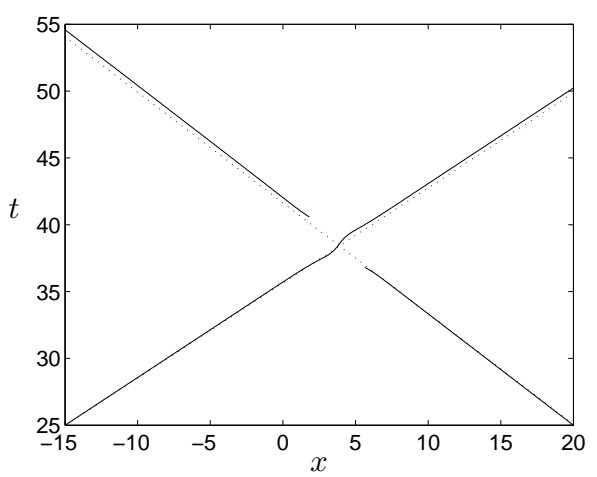

(d)

Fig. 2. Head-on collision of two $\eta$-solitary waves of unequal heights, $\theta^{2}=1$. (a) Interaction on the $x, t$ plane, (b) solution profile, $t=100$, (c) dispersive tails, $t=100$, (d) paths of the solitary waves in the $x, t$ plane.

solitary wave) appears to be monotonic. Qualitatively similar results are obtained from the headon collision of solitary waves of the same height. We summarize the results of the head-on collision experiments for the $\theta^{2}=1$ system in Table 3 . A qualitatively similar picture emerges from analogous experiments of head-on collisions of solitary waves of Bona-Smith systems with $2 / 3<\theta^{2}<1$.

Table 3

Head-on collision of solitary waves, $\theta^{2}=1, \eta$ profiles, $\longrightarrow$ : waves travelling to the right, $\longleftarrow$ : waves travelling to the left. Shown are the amplitude $(A)$ and speed $\left(c_{s}\right)$ of the colliding solitary waves before and well after the interaction, the magnitude of the phase shifts of the emerging waves at $t=80$, and the maximum amplitude of the solution during the interaction.

\begin{tabular}{|c|c|c|c|c|c|c|c|c|c|c|}
\hline \multicolumn{4}{|c|}{ Before } & \multicolumn{6}{|c|}{ After } & \multirow{2}{*}{$\begin{array}{l}\text { Maximum } \\
\text { amplitude }\end{array}$} \\
\hline \multicolumn{2}{|l|}{$\longrightarrow$} & \multicolumn{2}{|l|}{$\longleftarrow$} & \multicolumn{3}{|c|}{$\longrightarrow$} & \multicolumn{3}{|c|}{$\longleftarrow$} & \\
\hline$A$ & $c_{s}$ & $A$ & $c_{s}$ & $A$ & $c_{s}$ & ph. shift & $A$ & $c_{s}$ & ph. shift & \\
\hline 0.866190 & $1.4 \mid \mathrm{C}$ & 0.412617 & 1.2 & 0.865921 & 1.39988 & 0.3194 & 0.412543 & 1.19994 & 0.5102 & 1.384417 \\
\hline 0.866190 & 1.4 & symmet & & 0.865536 & 1.39972 & 0.4753 & & ymmetri & & 1.926950 \\
\hline 0.412617 & 1.2 & symmet & & 0.412572 & 1.19996 & 0.3440 & & ymmetri & & 0.881577 \\
\hline
\end{tabular}




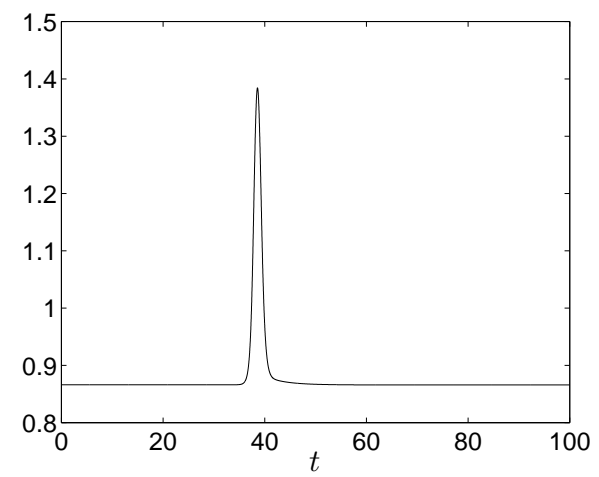

(a)

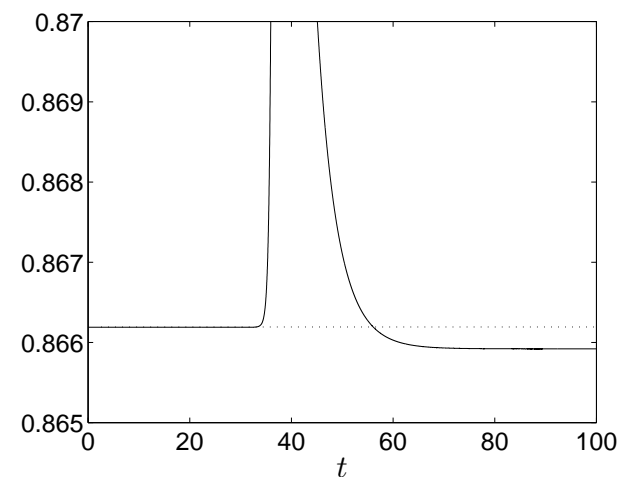

(b)

Fig. 3. Maximum amplitude of $\eta$ during the collision of Figure 2; (b) is a magnification of (a).

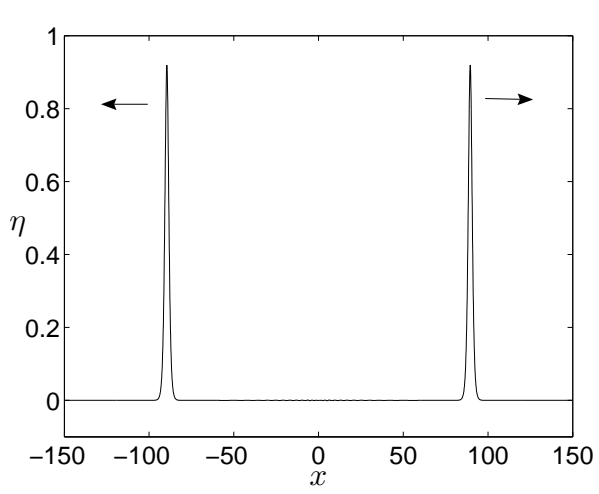

(a)

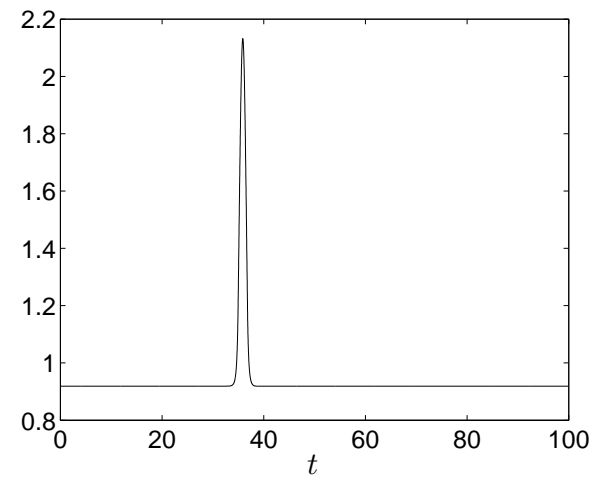

(c)

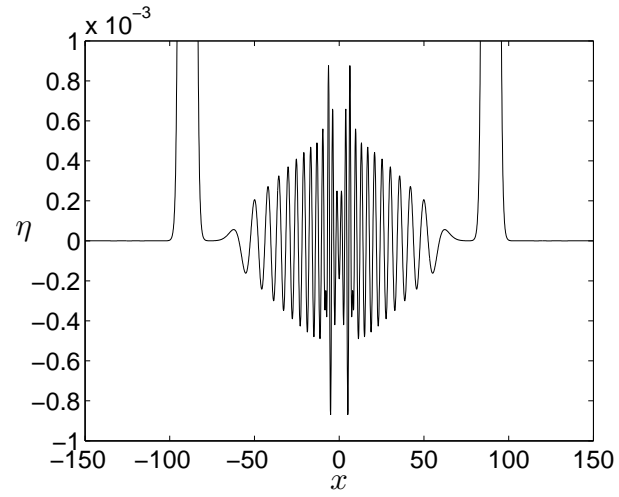

(b)

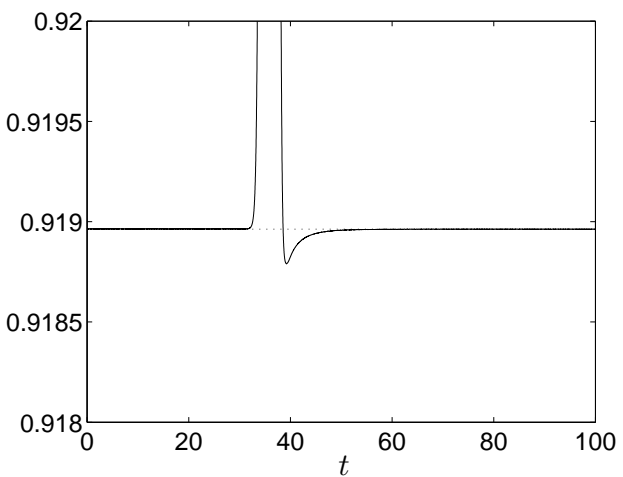

(d)

Fig. 4. Head-on collision of two $\eta$-solitary waves of equal heights, $\theta^{2}=2 / 3$. (a) Solution profile after the interaction, $t=100$, (b) dispersive tails, $t=100$, (c) maximum amplitude of $\eta$ during the head-on collision, (d) a magnification of (c). 
Table 4

Head-on collision of solitary waves, $\theta^{2}=2 / 3, \eta$ profiles. Labels as in Table 3 .

\begin{tabular}{|c|c|c|c|c|c|c|c|c|c|c|}
\hline \multicolumn{4}{|c|}{ Before } & \multicolumn{6}{|c|}{ After } & \multirow{2}{*}{$\begin{array}{l}\text { Maximum } \\
\text { amplitude }\end{array}$} \\
\hline \multicolumn{2}{|l|}{$\longrightarrow$} & \multicolumn{2}{|l|}{$\longleftarrow$} & \multicolumn{3}{|c|}{$\longrightarrow$} & \multicolumn{3}{|c|}{$\longleftarrow$} & \\
\hline$A$ & $c_{s}$ & $A$ & $c_{s}$ & $A$ & $c_{s}$ & ph. shift & $A$ & $c_{s}$ & ph. shift & \\
\hline 0.918963 & 1.4 & 0.429752 & 1.2 & 0.918952 & 1.399995 & 0.3294 & 0.429756 & 1.200001 & 0.5141 & 1.498639 \\
\hline 0.918963 & 1.4 & symmetr & & 0.918962 & 1.4 & 0.4805 & & ymmetric & & 2.131893 \\
\hline 0.429752 & 1.2 & symmetr & & 0.429752 & 1.2 & 0.3526 & & ymmetric & & 0.936017 \\
\hline
\end{tabular}

In the case of the BBM-BBM system $\left(\theta^{2}=2 / 3\right)$ the results of our few head-on collision experiments are summarized in Table 4 and are in good agreement with the much richer collection of numerical results in [6]. When two solitary solitary waves of different heights, specifically of initial $\eta$-amplitudes $A_{1}=0.918963, A_{2}=0.429752$ and speeds $c_{s, 1}=1.4, c_{s, 2}=1.2$, were let to collide head-on, we observed that, well after the collision, the longer wave acquired the slightly smaller amplitude $A_{1}^{\prime}=0.918952$, while the smaller one grew slightly to $A_{2}^{\prime}=0.429756$. During two similar experiments for waves of the same height (initially equal to 0.918963 and 0.429752 ) we observed that, well after the collision, the waves returned to practically their initial amplitudes and speeds. This may be seen in the graph of the evolution of the total amplitude during the head-on collision of two waves of initial amplitude 0.918963 in Figure 4(d). Note that this transition in the temporal evolution of the maximum amplitude is no longer monotonic: We first observe a transient loss of amplitude and then a gradual increase to the original amplitude of the larger wave, in agreement with the analogous result of [6]. In contrast to this behaviour, it was previously seen that the analogous numerical experiments with Bona-Smith systems with $\theta^{2} \neq 2 / 3$ show a monotonic decrease to a permanent loss of amplitude. It is known, cf. e.g. [29], that experimentally observed solitary waves reflected from a vertical wall exhibit a permanent loss of amplitude, but this could be due to a large extent to dissipative effects present in any experiment in real channels with real fluids. It is worth note that analogous numerical simulations of the Euler equations, cf. [16] and the references quoted therein, indicate that the maximum amplitude returns slowly to its initial value.

Finally, note also the much smaller size of the dispersive tails in Figures 4(a) and (b) relative to the analogous sizes of dispersive tails of other Bona-Smith systems. Another difference is that the tails still interact at $t=100$ and have not separated; for an explanation of this phenomenon cf. [18].

\subsubsection{Overtaking collisions}

We proceed now to describe the outcome of some numerical experiments simulating overtaking collisions of two solitary waves propagating in the same direction. In the case of one-way models, this type of interaction has been studied in detail analytically by the inverse scattering transform in the case of integrable equations like the KdV, cf. e.g. [24], and numerically in the case of nonintegrable models, cf. e.g. [11]. It has also been studied experimentally and numerically in the case of the full Euler equations; we refer the reader to [16] for a recent detailed study and review of the literature.

We consider first the Bona-Smith system with $\theta^{2}=9 / 11$, which we integrate on $[-400,400]$ with periodic boundary conditions using $h=0.05$ and $k=0.005$. Two solitary waves of $\eta$-amplitudes equal to $A_{1}=0.8912762$ and $A_{2}=0.4214631$ are initially centered at $x=-40$ and $x=40$, and given initial speeds equal to $c_{1}=1.4$ and $c_{s}=1.2$, respectively. As they travel to the right, the larger and faster wave overtakes the smaller and slower one, they interact from about $t=350$ to $t=420$, emerge basically unchanged, and continue moving to the right, cf. Figure 5 . The interaction process is much slower than the head-on collision, as expected. The emerging large solitary wave has now 

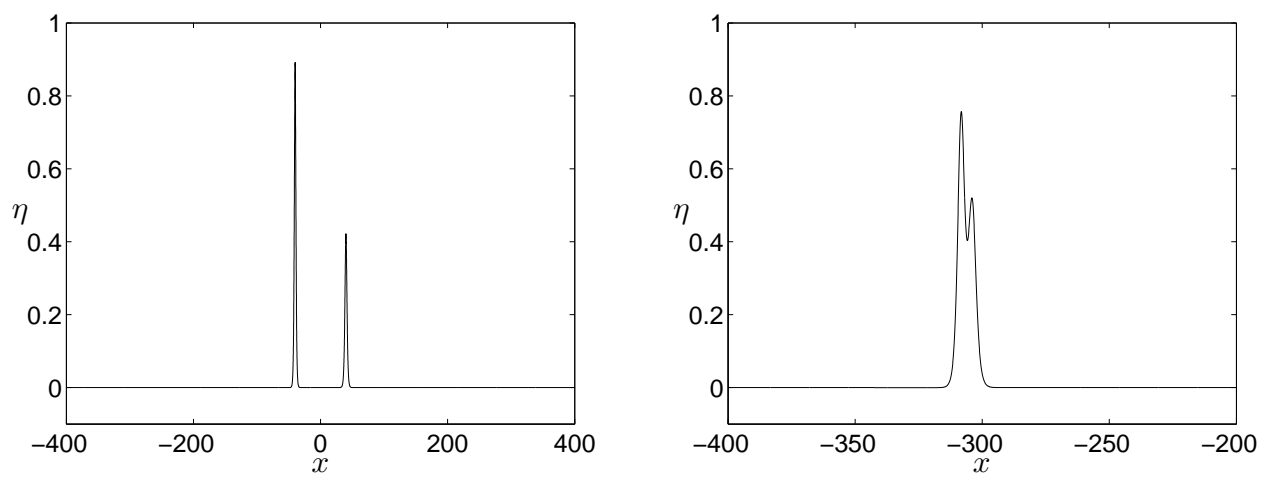

$t=0$

$$
t=380
$$
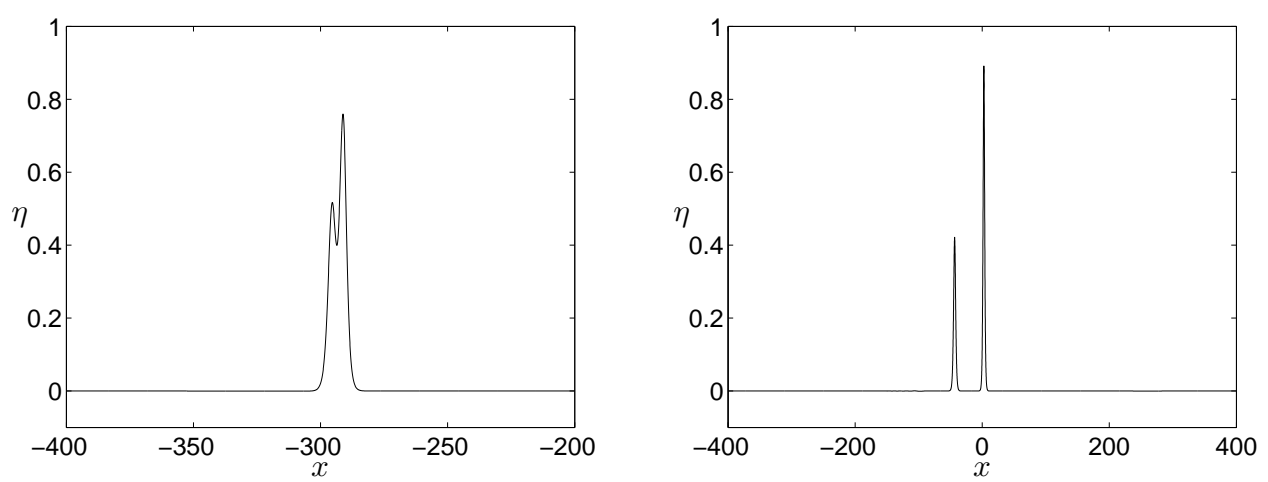

$t=390$

$$
t=600
$$

Fig. 5. Overtaking collision of two $\eta$-solitary waves, $\theta^{2}=9 / 11$.

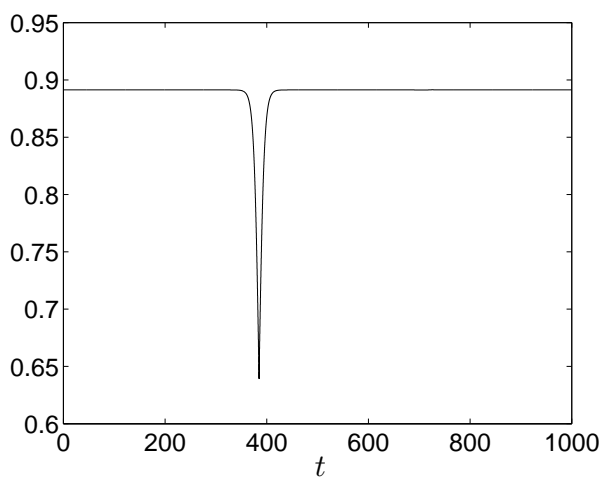

(a)

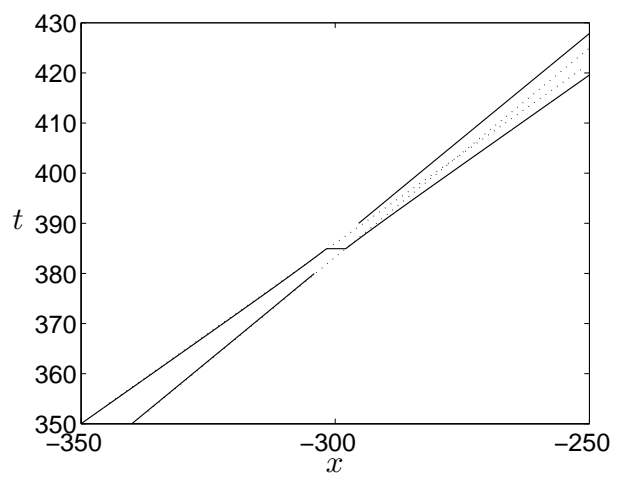

(b)

Fig. 6. Overtaking collision of two solitary waves, $\theta^{2}=9 / 11$. (a) Maximum value of $\eta$ as function of $t$, (b) paths of the solitary waves in the $x, t$ plane. 

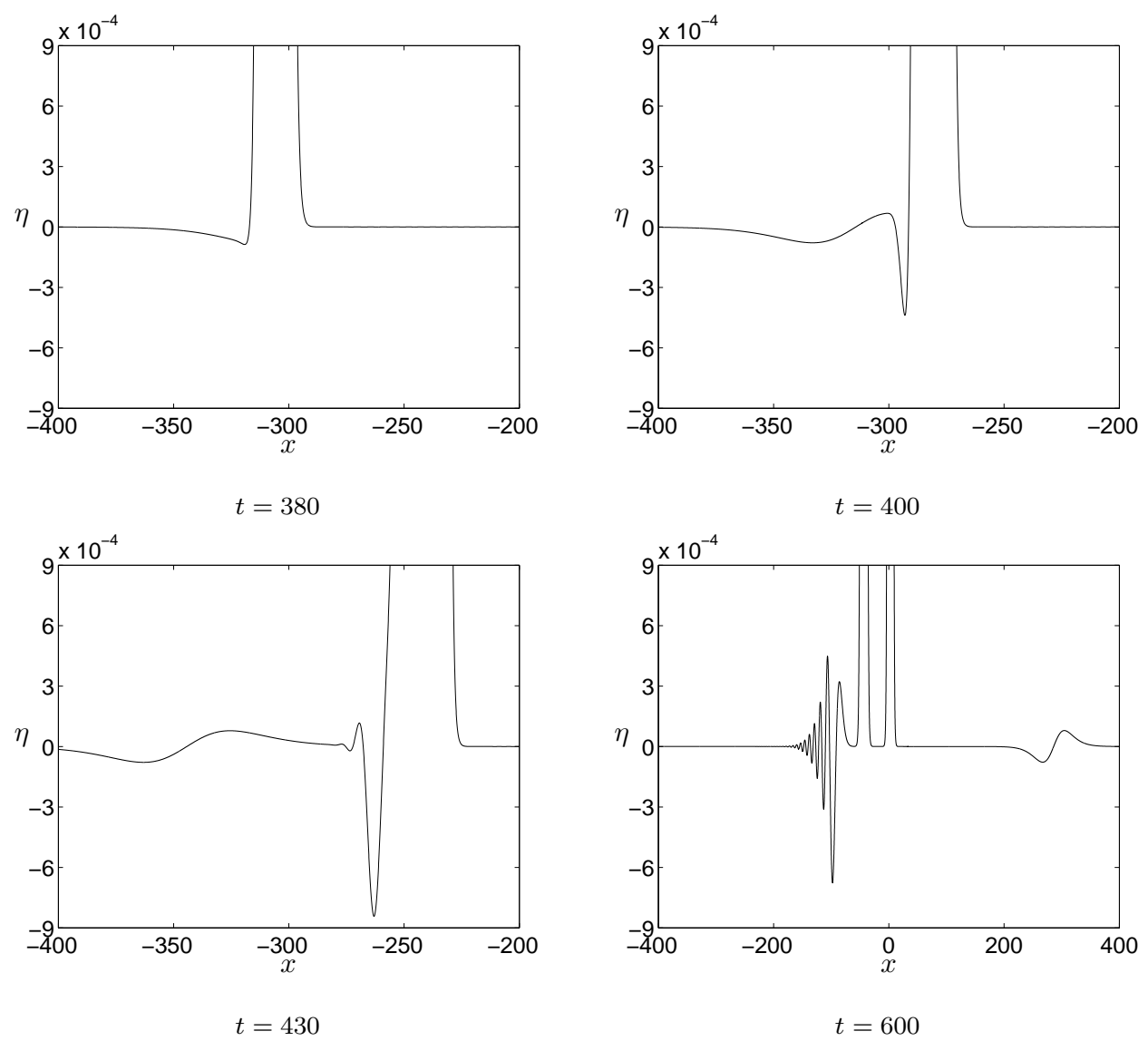

Fig. 7. Dispersive oscillations produced by the overtaking collision of Figure 5.

a slightly larger amplitude $A_{1}^{\prime}=0.8912769$, while the small one has a slightly smaller amplitude equal to $A_{2}^{\prime}=0.4214596$. The speeds of the emerging waves are found to be equal to 1.4 and 1.2 again, i.e. to return to their initial values within the numerical accuracy of our computations. It is worthwhile to note that during this type of collision the maximum value of $\eta$ during the interaction dips below the initial amplitude of the larger solitary wave but remains larger than the amplitude of the smaller wave (cf. Figure 6(a)), in contrast to what happens in head-on collisions, where the maximum value exceeds the sum of the heights of the two waves. The two waves undergo relatively large phase changes during the interaction, see Fig. 6(b), with the larger solitary wave gaining in phase and the smaller being delayed. For example, at $t=420$ the phase shifts are equal to about 2.62 and 3.41 spatial units for the larger and smaller waves, respectively. These observations are in qualitative agreement with the well-known analogous facts about overtaking collisions in the case of the KdV, the BBM and the Euler equations, cf. e.g. [24], [11], [16].

The overtaking collision depicted in the sequence of plots of Figure 5 is not elastic. Magnifying the $\eta$-axis reveals that the interaction produces small-amplitude dispersive oscillations; these magnified profiles are shown at four temporal instances in Figure 7 . The profiles at $t=380,400,430$ show the early stages of generation of these oscillations, while the profile at $t=600$ shows their full development. They consist of two types of tiny waves: A dispersive tail, resembling the dispersive tails generated by head-on collisions, moves to the right and follows the solitary waves. Being slower than the smaller solitary wave, the tail is already observed at $t=600$ to have detached itself from 


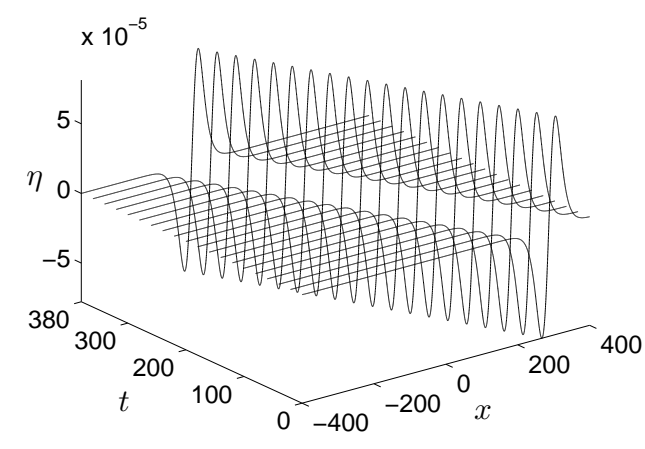

(a)

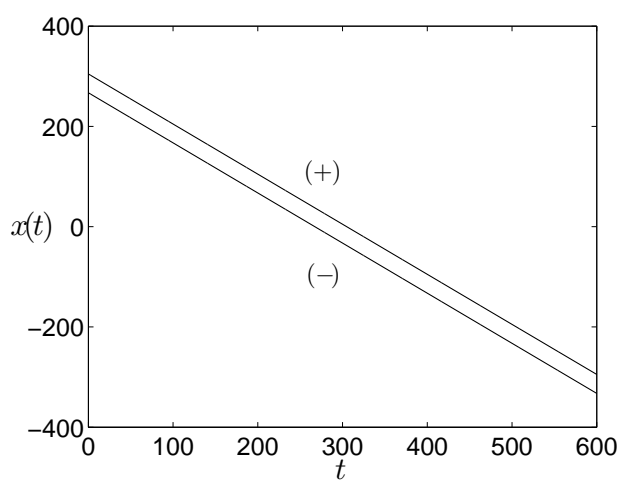

(c)

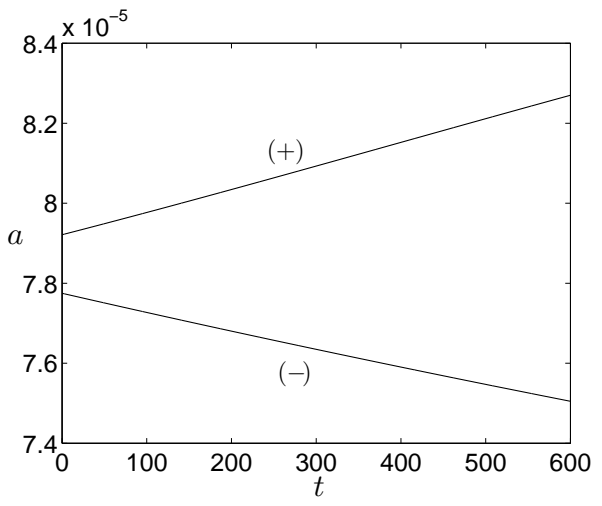

(b)

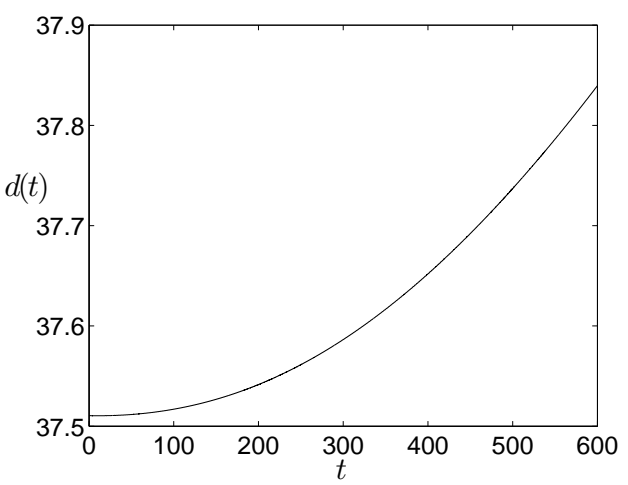

(d)

Fig. 8. Wavelet produced by the overtaking collision of Figures 5 and 7 . (a) Perspective of the evolution, (b) absolute amplitude of the peaks of the wavelet. (c) Paths of the peaks in the $x, t$ plane, (d) distance of peaks as a function of $t$.

the latter. In addition, a small wavelet, resembling an inverted $N$, is generated and travels to the left. (By $t=600$ it has wrapped itself around the endpoint by periodicity.)

This picture is in good qualitative agreement with the outcome of numerical simulations of overtaking collisions of solitary waves of the Euler equations in [16]. The small wavelet structure moving to the left was identified by $\mathrm{Su}$ and Zou in [33] in the course of numerical simulations of overtaking collisions of solitary waves of a fourth-order Boussinesq-type equation approximating the Euler equations. However this equation incorporates a one-way assumption and does not properly model two-way surface wave propagation.

To study further the wavelet numerically, we isolate it from the rest of the solution by setting $\eta$ equal to zero at $t=600$ in the interval [-230.85,25.25] containing the solitary waves and the dispersive tail (cf. Figure 7). We use the cleaned wavelet as new initial condition resetting $t=0$, furnish it with its (cleaned) velocity profile, and let it travel to the left. Figure 8(a) shows the ensuing evolution of the wavelet, which gives the impression that it is moving at a constant speed without change of shape. This is not true however. Our computations show that the amplitude of the positive peak $(+)$ is apparently slowly increasing, while the absolute amplitude of the negative peak $(-)$ is slowly decreasing over a temporal interval $[0,600]$ of integration, cf. Figure $8(\mathrm{~b})$. The paths $x(t)$ of the two peaks are shown in Figure 8(c), and their absolute difference is plotted in (d). 
It seems that the speeds of the peaks vary slowly with $t$, both being approximately equal to 1 in absolute value. Specifically, the absolute value of the speed of $(+)$ decreases form 0.999129 at $t=20$ to 0.998237 at $t=600$, while that of $(-)$ increases from 0.999143 at $t=20$ to 0.999326 .

We observed qualitatively similar evolutions in numerical experiments with overtaking collisions of solitary waves of other Bona-Smith systems. It might be worthwhile to note that for $\theta^{2}$ close to $2 / 3$ the left-travelling small wavelet became $N$-shaped, i.e. the positive excursion preceded the negative one.

\subsection{Interaction of solitary waves with the boundary}

In this section we present the results of some numerical experiments illustrating interactions of solitary waves of the Bona-Smith systems with the endpoints of an interval, where either reflection or homogeneous Dirichlet boundary conditions for $\eta$ and $u$ have been postulated. (All computations in this section were done with $h=0.1, k=0.01$.)

The ideal reflection of a solitary wave at a boundary point (where $\eta_{x}=0, u=0$ ) is equivalent to the head-on collision of the solitary wave with its symmetric image about this point. This is illustrated in the sequence of plots of Figure 9. An exact solitary wave of the system corresponding to $\theta^{2}=9 / 11$ of unit $\eta$-amplitude (computed by the formulas (29)-(30) and initially centered at $x=$ $100)$ is approximated numerically as it travels to the right and hits, at about $t=35$, the boundary $x=150$, where reflection boundary conditions have been assumed to hold. The $\eta$-component of this wave is represented by the solid line in Figure 9. The solitary wave is observed to be reflected backwards and a trailing dispersive tail is generated. Another experiment is also shown superimposed in the same figure. The dotted line represents an identical $\eta$-solitary wave initially placed at $x=200$ and given an opposite velocity. If there were no boundary the two waves would collide head-on at $x=150$. This collision is also shown in Figure 9: The two waves collide, (the maximum amplitude of this interaction is achieved at $t=35$ ) and continue travelling in their respective directions, having suffered a slight phase shift and amplitude and speed reduction as was seen in the previous section. (The waves are still denoted by a dotted line but the wave travelling to the left coincides to graph thickness with the reflected wave.)

We now turn to a series of experiments illustrating what happens when a solitary wave hits a boundary where homogeneous Dirichlet boundary conditions for $\eta$ and $u$ are assumed to hold. Figure 10 shows a $\eta$-solitary wave of the Bona-Smith system with $\theta^{2}=9 / 11$ of amplitude $A=1$ (computed again by the exact formulas, so that its speed is $c_{s}=5 \sqrt{3} / 6 \cong 1.44337$ ) travelling to the right until it hits the boundary $x=150$, where $\eta=u=0$. Apparently, the wave is 'reflected' backwards and small-amplitude dispersive oscillations seem to be generated at the boundary and to spread leftwards with a front that travels slower than the main pulse. The main pulse is a solitary wave and stabilizes at the slightly larger amplitude $A^{\prime}=1.000660$ and speed $c_{s}^{\prime}=1.44363$. The time history of the amplitude of $\eta$ up to $t=300$ is shown in Figure 11(a). Figure 11(b) is a magnification of 11(a) around the interaction time. Figure 11(c) shows the behaviour of the (spatial) $L^{2}$-norm of $\eta$ as a function of $t$. Note the transient nonmonotonic behaviour of the amplitude of $\eta$ during the interaction, before it stabilizes to its higher value, and the momentary dip of the $L^{2}$ norm. A similar eventual increase is observed in the analogous quantities (absolute amplitude and $L^{2}$ norm) of the $u$ component of the solution. Thus, in the case of the $\theta^{2}=9 / 11$ system, in contrast to the reflection of a solitary wave at an ideal reflection boundary $\left(\eta_{x}=u=0\right)$, which is equivalent to a collision of two solitary waves symmetric about the boundary and where, therefore, the height of the wave reflected is decreased, a homogeneous Dirichlet boundary condition on $\eta$ and $u$ seems to return a larger wave.

A similar behaviour was observed when a solitary wave of the $\theta^{2}=1$ system interacted with 

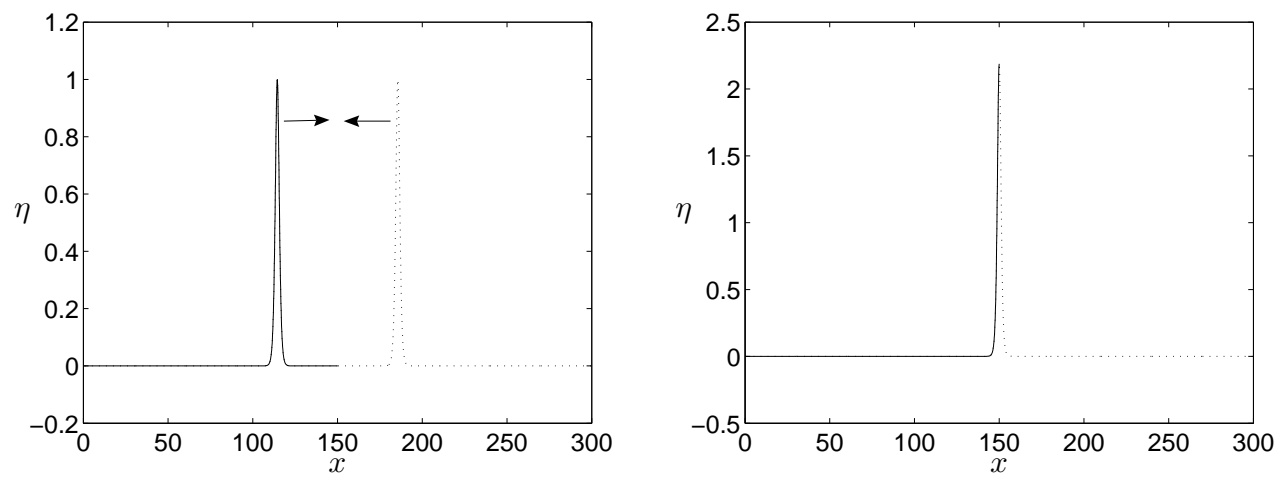

$\eta$ at $t=10$

$\eta$ at $t=35$
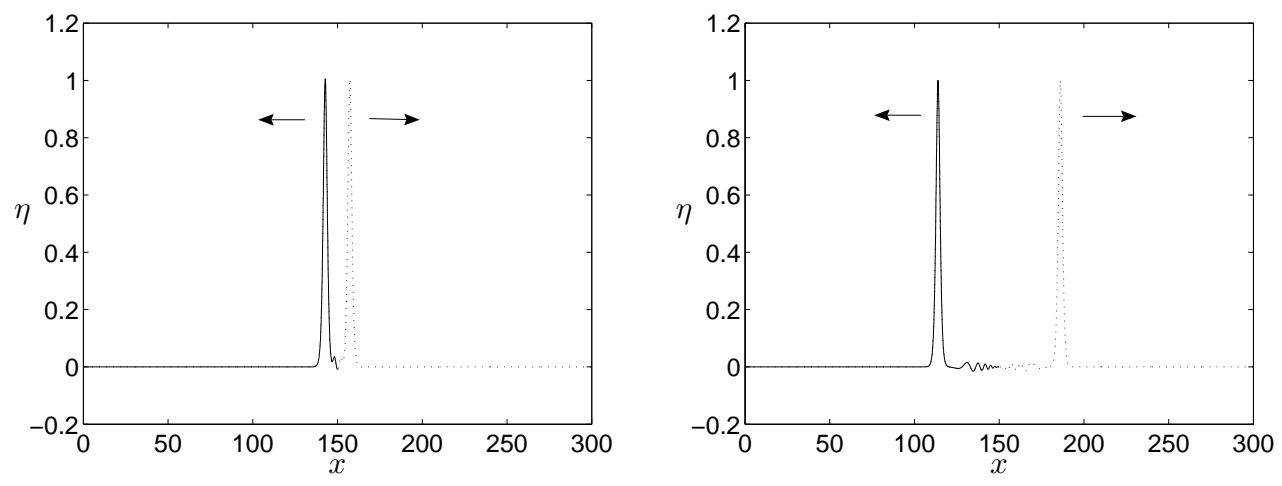

$\eta$ at $t=40$

$\eta$ at $t=60$

Fig. 9. Reflection of an $\eta$-solitary wave at $x=150, \theta^{2}=9 / 11$. Solid line: reflected solitary wave. Dotted line: two $\eta$-solitary waves of equal height colliding. After the collision the leftwards-travelling wave coincides, within graph thickness, with the reflected wave.
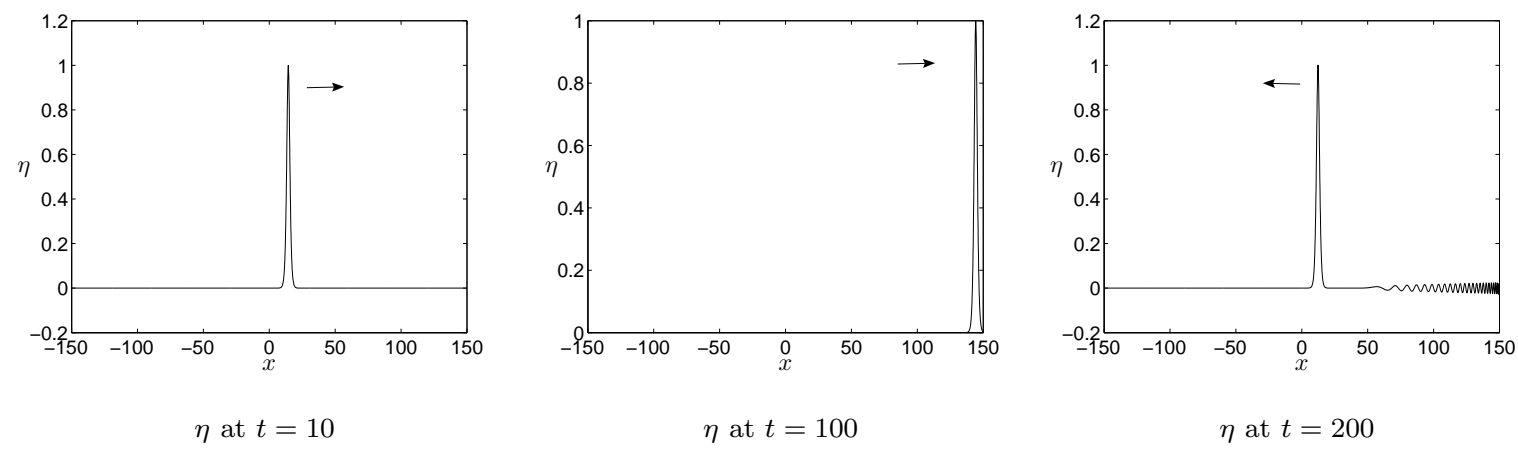

Fig. 10. Interaction of a solitary wave with the boundary $\eta=u=0$ at $x=150, \theta^{2}=9 / 11$

a boundary where homogeneous Dirichlet boundary conditions were posed. Indeed, an $\eta$-solitary wave of initial amplitude $A=0.866190$ is 'reflected' backwards from such a boundary with a larger amplitude, which stabilizes to the value $A^{\prime}=0.868607$. However, as the other end of the interval $[2 / 3,1]$ of $\theta^{2}$ values is approached, the behaviour of the wave that is returned backwards changes. 


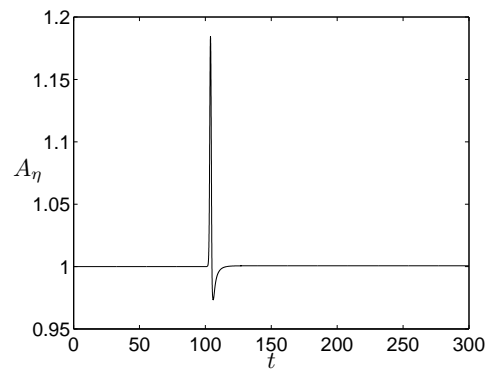

(a)

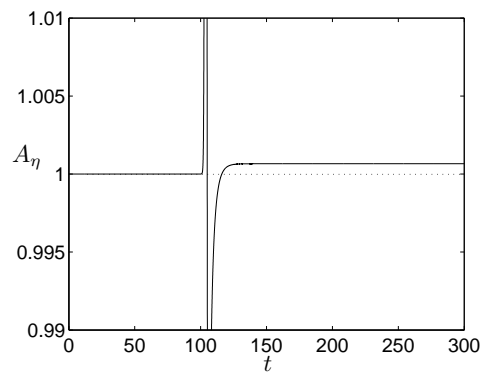

(b)

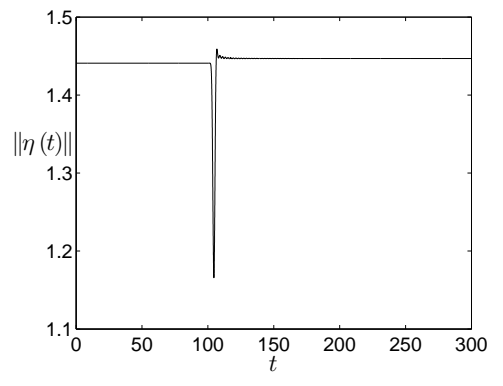

(c)

Fig. 11. Evolution of Figure 10. (a) Amplitude of $\eta$ as a function of $t$, (b) a magnification of (a), (c) $L^{2}$ norm of $\eta$ as a function of $t$.

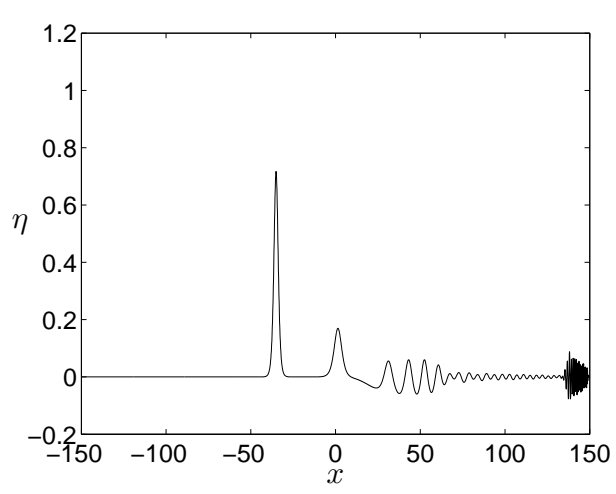

(a)

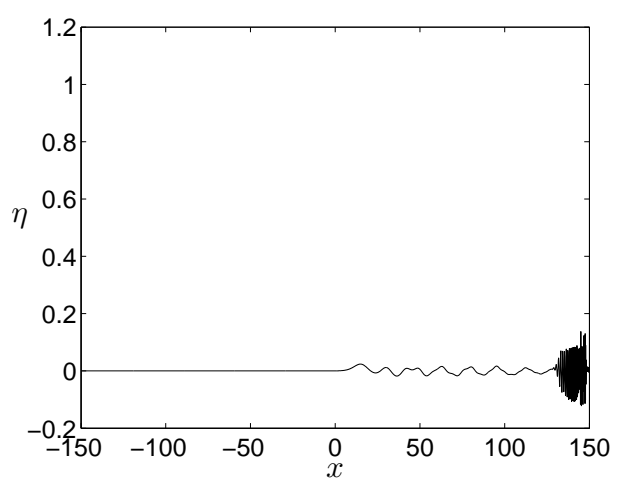

(b)

Fig. 12. Interaction of a solitary wave with the boundary $\eta=u=0$ at $x=150$. 'Reflected' wave at $t=250$ : (a) $\theta^{2}=2 / 3+0.01$, (b) $\theta^{2}=2 / 3$.

For example, Figure 12(a) shows the $\eta$-profile at $t=250$ of the solution of the system corresponding to $\theta^{2}=2 / 3+0.01$ that emanates from a single solitary wave initially centered at $x=0$ with a height $A=0.916990$. The wave travels to the right, hits the Dirichlet boundary at $x=150$ and returns, as Figure 12(a) shows, as a wavetrain composed by a main solitary wave pulse of smaller height $A^{\prime}=0.717377$ apparently followed by a smaller solitary wave and dispersive oscillations, a high-frequency group of which stays close to the boundary. When the experiment is repeated with a solitary wave of the BBM-BBM $\left(\theta^{2}=2 / 3\right)$ system of initial height equal to 0.918963 , no solitary wave is visible at $t=250$ in the returning oscillations, cf. Figure $12(\mathrm{~b})$, while the high-frequency group, near the boundary has larger amplitude. It appears that the BBM-BBM system is singular in this respect as well.

The fact that for values of $\theta^{2}$ near 1 the solitary wave that returns from the right-hand Dirichlet boundary is larger that the impinging one raises the natural question whether it will keep growing after repeated collisions at both endpoints of the spatial interval of integration where homogeneous Dirichlet b.c.'s hold. In the numerical experiment that yielded the results of Figure 13, a solitary wave of unit initial amplitude $\left(\theta^{2}=9 / 11\right)$ travels to the right at first and is 'reflected' back and forth for $0 \leq t \leq 500$, suffering repeated 'collisions' at the boundaries $x= \pm 50$, where $\eta=u=0$. After each 'collision' (cf. also Figure 11(c)) the mean level of the $L^{2}$ norm of $\eta$ increases, but the norm oscillates due to the increased presence of the boundary-generated oscillations that grow as 


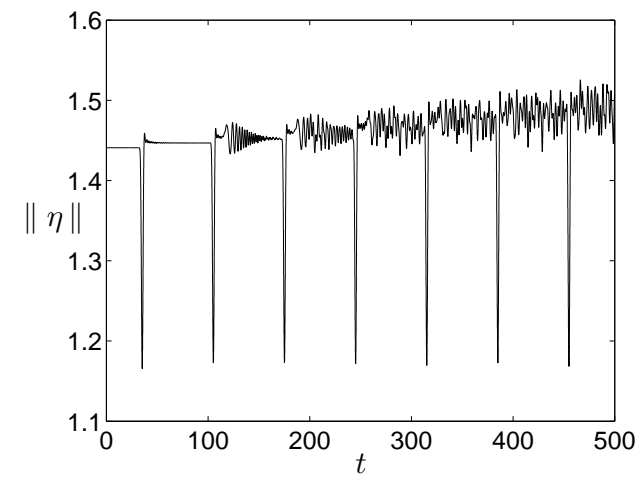

(a)

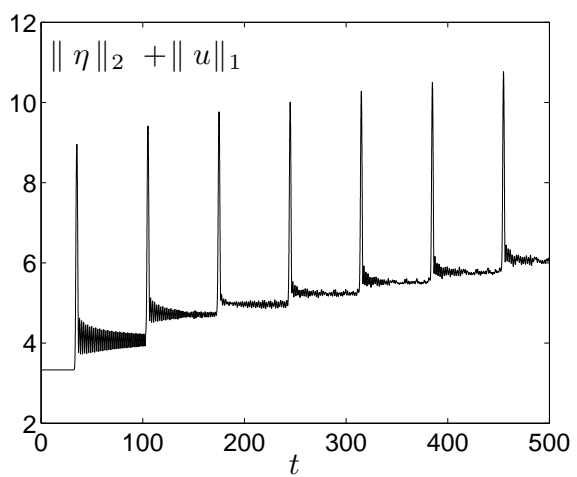

(b)

Fig. 13. Ping-pong of a solitary wave between $x= \pm 50$, where $\eta=u=0, \theta^{2}=9 / 11$. (a) $\|\eta\|$ as a function of $t$, (b) $\|\eta\|_{2}+\|u\|_{1}$ as a function of $t$.

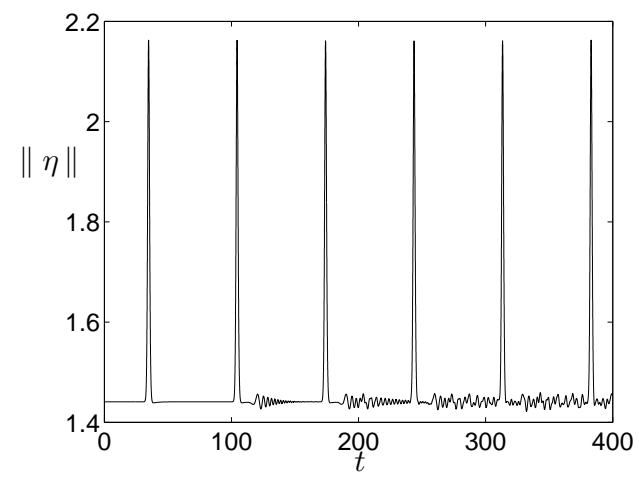

(a)

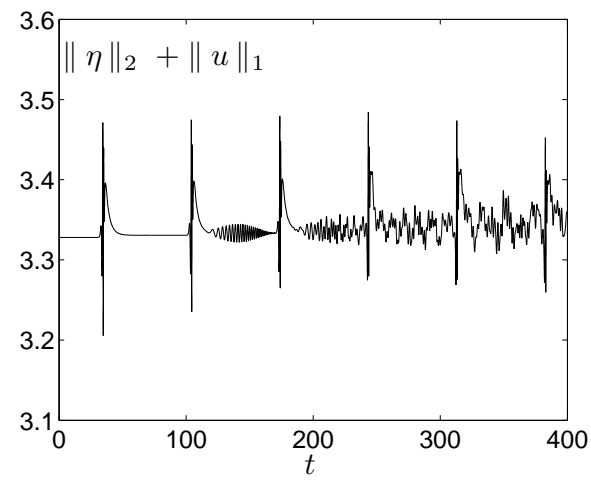

(b)

Fig. 14. Ping-pong of a solitary wave between $x= \pm 50$, where $\eta_{x}=u=0, \theta^{2}=9 / 11$. (a) $\|\eta\|$ as a function of $t$, (b) $\|\eta\|_{2}+\|u\|_{1}$ as a function of $t$.

they interact with themselves and with the solitary wave. A slow growth of the $\|\eta\|_{2}+\|u\|_{1}$ norm with time is clearly observed. (In [4] it was proved that the ibvp with Dirichlet boundary conditions is locally well-posed in $H^{2} \times H^{1}$. The spikes in both graphs represent momentary changes of the norms at 'collision' times.).

This behaviour is to be contrasted with that of the analogous experiment with perfect reflection (i.e. $\eta_{x}=u=0$ ) at $x= \pm 50$, cf. Figure 14. Between collisions, the quantities $\|\eta\|$ and $\|\eta\|_{2}+\|u\|_{1}$ oscillate around a practically constant mean level. (This ibvp has been shown in [4] to possess a unique classical solution in $[0, T]$ for any $T<\infty$, under mild restrictions on the initial data.)

\section{Acknowledgment}

The authors record their thanks to Jerry Bona for many discussions and suggestions on the Boussinesq systems over the years. D. Mitsotakis was supported by Marie Curie Fellowship No. PIEF-GA-2008-219399 of the European Commission. 


\section{References}

[1] D. C. Antonopoulos, The Boussinesq system of equations: Theory and numerical analysis, Ph.D. Thesis, University of Athens, in Greek (2000).

[2] D. C. Antonopoulos, V. A. Dougalis, Galerkin methods for the Bona-Smith version of the Boussinesq equations, in: P. S. Theocaris (ed.), Proceedings of the Fifth National Congress on Mechanics, 1998, pp. 1001-1008, Ioannina, Greece.

[3] D. C. Antonopoulos, V. A. Dougalis, Numerical approximations of Boussinesq systems, in: A. Bermudez, D. Gomez, C. Hazard, P. Joly, J. Roberts (eds.), Proceedings of the 5th International Conference on Mathematical and Numerical Aspects of Wave Propagation, SIAM, Philadelphia, 2000, pp. 265-269.

[4] D. C. Antonopoulos, V. A. Dougalis, D. E. Mitsotakis, Initial-boundary-value problems for the Bona-Smith family of Boussinesq systems, Advances in Differential Equations 14 (2009) 27-53.

[5] G. Baker, V. A. Dougalis, O. A. Karakashian, Convergence of Galerkin approximation for the Korteweg-deVries equation, Math. Comp. 40 (1983) 419-433.

[6] J. L. Bona, M. Chen, A Boussinesq system for two-way propagation of nonlinear dispersive waves, Physica D 116 (1998) 191-224.

[7] J. L. Bona, M. Chen, J.-C. Saut, Boussinesq equations and other systems for small-amplitude long waves in nonlinear dispersive media: I. Derivation and Linear Theory, J. Nonlinear Sci. 12 (2002) 283-318.

[8] J. L. Bona, M. Chen, J.-C. Saut, Boussinesq equations and other systems for small-amplitude long waves in nonlinear dispersive media: II. The nonlinear theory, Nonlinearity 17 (2004) 925-952.

[9] J. L. Bona, V. A. Dougalis, O. A. Karakashian, W. R. McKinney, Conservative high-order numerical schemes for the generalized Korteweg-deVries equation, Phil. Trans. R. Soc. London A 351 (1995) 107-164.

[10] J. L. Bona, V. A. Dougalis, D. E. Mitsotakis, Numerical solution of KdV-KdV systems of Boussinesq equations I. The numerical scheme and generalized solitary waves, Math. Comp. Simul. 74 (2007) 214-228.

[11] J. L. Bona, W. G. Pritchard, L. R. Scott, Solitary-wave interaction, Phys. Fluids 29 (1980) 438-441.

[12] J. L. Bona, R. Smith, A model for the two-way propagation of water waves in a channel, Math. Proc. Camb. Phil. Soc. 79 (1976) 167-182.

[13] P. Chatzipantelidis, Explicit multistep methods for nonstiff partial differential equations, Appl. Numer. Math. 27 (1998) 13-31.

[14] M. Chen, Exact traveling-wave solutions to bi-directional wave equations, Int. J. Theor. Phys. 37 (1998) $1547-$ 1567.

[15] M. Chen, Solitary-wave and multipulsed traveling-wave solutions of Boussinesq systems, Applicable Analysis 75 (2000) 213-240.

[16] W. Craig, P. Guyenne, J. Hammack, D. Henderson, C. Sulem, Solitary water wave interactions, Phys. Fluids 18 (2006) 57-106.

[17] C. DeBoor, G. J. Fix, Spline approximation by quasiinterpolants, J. Approximation Theory 8 (1973) $19-45$.

[18] V. A. Dougalis, A. Duran, M. A. Lopez-Marcos, D. E. Mitsotakis, A numerical study of the stability of solitary waves of the Bona-Smith system, J. Nonlinear Sci. 17 (2007) 569-607.

[19] V. A. Dougalis, O. A. Karakashian, On some high-order accurate fully discrete Galerkin methods for the KortewegdeVries equation, Math. Comp. 45 (1985) 329-345.

[20] V. A. Dougalis, D. E. Mitsotakis, Solitary waves of the Bona-Smith system, in: D. Fotiadis, C. Massalas (eds.), Advances in scattering theory and biomedical engineering, World Scientific, 2004, pp. 286-294.

[21] V. A. Dougalis, D. E. Mitsotakis, Theory and numerical analysis of Boussinesq systems: A review, in: N. A Kampanis, V. A. Dougalis, J. A. Ekaterinaris (eds.), Effective Computational Methods in Wave Propagation, CRC Press, 2008, pp. 63-110.

[22] T. Dupont, Galerkin methods for first order hyperbolics: an example, SIAM J. Numer. Anal. 10 (1973) 890-899.

[23] J. D. Fenton, A ninth-order solution for the solitary wave, J. Fluid Mech. 53 (1972) 257-271.

[24] P. D. Lax, Integrals of nonlinear equations of evolution and solitary waves, Commun. Pure Appl. Math. 21 (1968) 467-490.

[25] D. E. Mitsotakis, Theory and numerical analysis of nonlinear dispersive wave equations: Boussinesq systems in one and two dimensions, Ph.D. Thesis, University of Athens, in Greek (2007).

[26] R. L. Pego, M. I. Weinstein, Convective linear stability of solitary waves for the Boussinesq equations, Studies Appl. Math. 99 (1997) 311-375.

[27] B. Pelloni, Spectral methods for the numerical solution of nonlinear solution dispersive wave equations, Ph.D. Thesis, Yale University (1996).

[28] B. Pelloni, V. A. Dougalis, Numerical modelling of two-way propagation of nonlinear dispersive waves, Math. Comput. Simul. 55 (2001) 595-606.

[29] D. P. Renouard, F. J. S. Santos, A. M. Temperville, Experimental study of the generation, damping, and reflexion of a solitary wave, Dynamics of Atmospheres and Oceans 9 (1985) 341-358. 
[30] J. S. Russell, Report on waves, 14th Meeting of the British Association for the Advancement of Science, John Murray, London, 1844, pp. 311-390.

[31] R. Schreiber, Finite element methods of high-order accuracy for singular two-point boundary value problems with nonsmooth solutions, SIAM J. Numer. Anal. 17 (1980) 546-566.

[32] C.-H. Su, R. M. Mirie, On head-on collisions between two solitary waves, J. Fluid Mech. 98 (1980) 509-525.

[33] C.-H. Su, Q.-S. Zou, Waves generated by collisions of solitary waves, Phys. Rev. A 35 (1987) 4738-4742.

[34] V. Thomée, L. B. Wahlbin, Maximum-norm stability and error estimates in Galerkin methods for parabolic equations in one space variable, Numer. Math. 41 (1983) 345-371.

[35] V. Thomée, B. Wendroff, Convergence estimates for Galerkin methods for variable coefficient initial value problems, SIAM J. Numer. Anal. 11 (1974) 1059-1068.

[36] J. F. Toland, Solitary wave solutions for a model of the two-way propagation of water waves in a channel, Math. Proc. Camb. Philos. Soc. 90 (1981) 343-360.

[37] J. F. Toland, Uniqueness and a priori bounds for certain homoclinic orbits of a Boussinesq system modelling solitary water waver, Commun. Math. Phys. 94 (1984) 239-254.

[38] J. F. Toland, Existence of symmetric homoclinic orbits for systems of euler-langrange equations, in: Proceedings of Symposia in Pure Mathematics, vol. 45, American Mathematical Society, Providence, 1986, pp. 447-459.

[39] R. Winther, A finite element method for a version of the Boussinesq equation, SIAM J. Numer. Anal. 19 (1982) $561-570$. 